\title{
A Study of the Degree of Fluorination in Regioregular Poly(3-hexylthiophene)
}

\section{Citation}

Blaskovits, J. Terence, Thomas Bura, Serge Beaupré, Steven A. Lopez, Carl Roy, Julio de Goes Soares, Adam Oh, et al. 2016. "A Study of the Degree of Fluorination in Regioregular Poly(3-Hexylthiophene)." Macromolecules 50 (1) (December 27): 162-174. doi:10.1021/ acs.macromol.6b02365.

\section{Published Version}

doi:10.1021/acs.macromol.6b02365

\section{Permanent link}

http://nrs.harvard.edu/urn-3:HUL.InstRepos:35164984

\section{Terms of Use}

This article was downloaded from Harvard University's DASH repository, and is made available under the terms and conditions applicable to Open Access Policy Articles, as set forth at http:// nrs.harvard.edu/urn-3:HUL.InstRepos:dash.current.terms-of-use\#OAP

\section{Share Your Story}

The Harvard community has made this article openly available.

Please share how this access benefits you. Submit a story.

\section{Accessibility}




\section{A Study of the Degree of Fluorination in Regioregular Poly(3-hexylthiophene)}

J. Terence Blaskovits ${ }^{1 \#}$, Thomas Bura ${ }^{1 \#}$, Serge Beaupré ${ }^{1}$, Steven A. Lopez ${ }^{2}$, Carl Roy ${ }^{1}$, Julio de Goes Soares ${ }^{2}$, Adam $\mathrm{Oh}^{2}$, Jesse Quinn ${ }^{3}$, Yuning $\mathrm{Li}^{3}$, Alán Aspuru-Guzik ${ }^{2}$, Mario Leclerc ${ }^{1 *}$ \# J.T.B. and T.B. contributed equally

[*] Prof. Mario Leclerc

1) Canada Research Chair on Electroactive and Photoactive Polymers, Department of Chemistry, Université Laval, Quebec City, Quebec, Canada

2) Department of Chemistry and Chemical Biology, Harvard University, Cambridge, Massachusetts, U.S.A.

3) Department of Chemical Engineering, University of Waterloo, Waterloo, Ontario, Canada E-mail : Mario.Leclerc@,chm.ulaval.ca 
For Table of Contents Use Only:

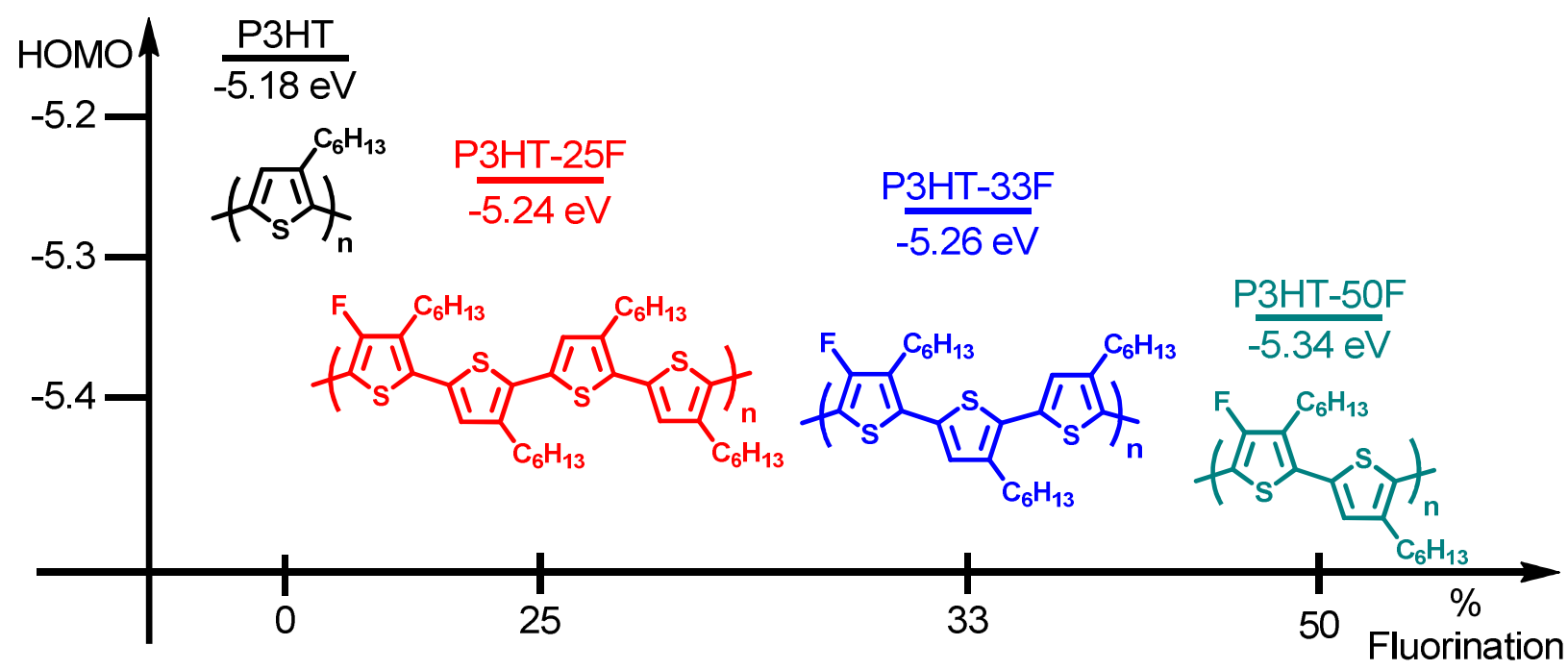

25

26

27

28

29

30

31

32

33

34

35

36

37

38

39

40

41

42

43

44

45

46

47

48

49

50

51

52

53

54

55

56

57

58

59

60 


\section{Abstract}

We systematically varied the degree of fluorination along the backbone of a series of highly regioregular 3-hexylthiophene-based polymers, P3HT-50F, P3HT-33F and P3HT-25F, in which $50 \%, 33 \%$ and $25 \%$ of the thiophene units within the polymer chain contain fluorine atoms in the available 4-position. These materials were homopolymerized using the Kumada catalyst transfer polycondensation method from a set of mono-fluorinated bi-, ter- and quarterthiophenes, to ensure high polymer regioregularity and evenly-spaced fluorine atoms along the conjugated thiophene backbone. The monomers were obtained from a synthetic route consisting of iterative Migita-Stille couplings of fluorinated and non-fluorinated 3hexylthiophenes. The effect of the fluorine atoms on both polymer structure and properties is presented, with supporting quantum mechanical calculations that rationalize the intrinsic conformation preferences of the three P3HT derivatives. P3HT-50F ( $\left.\bar{M}_{n}=34 \mathrm{~kg} / \mathrm{mol}, 98.5 \% \mathrm{rr}\right)$, P3HT-33F $\left(\bar{M}_{n}=46 \mathrm{~kg} / \mathrm{mol}, 98 \%\right.$ rr $)$ and P3HT-25F $\left(\bar{M}_{n}=53 \mathrm{~kg} / \mathrm{mol}, 95 \%\right.$ rr $)$ displayed HOMO levels of $-5.34,-5.26$ and $-5.24 \mathrm{eV}$, bandgaps of $1.98,1.98$ and $1.97 \mathrm{eV}$, and average field-effect transistor hole mobilities of $4.5 \times 10^{-3}, 2.7 \times 10^{-2}$, and $1.2 \times 10^{-2} \mathrm{~cm}^{2} \mathrm{~V}^{-1} \mathrm{~s}^{-1}$, respectively. 


\section{Introduction}

The impact of incorporating fluorine atoms into conjugated polymers has been the subject of much investigation. The incorporation of fluorine onto conjugated systems favors $\pi-\pi$ stacking, ${ }^{1}$, 2 and often results in a decrease of both the HOMO and LUMO frontier molecular orbital energies. ${ }^{3,4}$ The bandgap is thus constant, leading to a maintained absorption range of the material, while increasing the open-circuit voltage $\left(\mathrm{V}_{\mathrm{OC}}\right)$ in bulk heterojunction solar cells. ${ }^{5}$ The electronegativity of fluorine atoms can result in additional non-covalent interactions with adjacent repeating units which possess sulfur or hydrogen atoms in close spatial proximity to the fluorine moiety. These interactions promote coplanarity within a polymer chain, and strengthen inter- and intra-chain interactions. ${ }^{6}{ }^{7}$ However, the impact of fluorination on polymer morphology remains unpredictable, and both reduced solubility during polymerization and aggregation during device fabrication should be considered carefully when working with fluorinated homo- and copolymers. ${ }^{8}$ Fluorination at the 3-position of some thiophene-based units of conjugated polymers increases both the difficulty and cost of monomer synthesis, potentially limiting large-scale applications of these materials.

A number of studies have focused specifically on the preparation of fluorinated polythiophenes. These are summarized in Figure 1. Following initial reports of the electropolymerization of 3fluorothiophene ${ }^{9}$ and the synthesis of a perfluorinated oligothiophene ${ }^{10}$ in the early 2000 s, a patent describing different synthetic routes to obtain poly(3-alkylthiophene) derivatives with fluorinated backbones was presented. ${ }^{11}$ Drawing on these synthetic procedures, Roncali and colleagues have since described the electropolymerization of an alkylated mono-fluorinated terthiophene. ${ }^{12}$ Swager et al. also post-functionalized a brominated poly(3-hexylthiophene) at the 4-position using various electrophilic reagents. ${ }^{13}$ Using this method, the authors were able to 
incorporate fluorine atoms into $67 \%$ of the thiophene units using $N$-fluorobenzenesulfonimide (NFSI).

Two recently reported methods describe the synthesis of fluorinated thiophene monomers which were then polymerized using polycondensation techniques. First, the Kumada catalyst transfer polycondensation (KCTP) of 2,5-dibromo-3-fluoro-4-hexylthiophene resulted in a relatively regioregular (93\% regioregularity) fluorinated poly(3-hexylthiohene) (P3HT) by Heeney et al. ${ }^{14}$ However, the resulting polymer displayed very low solubility, and further characterization could not be undertaken. The authors then prepared analogue polymers with octyl and ethylhexyl side chains to improve solubility and better compare the effect of fluorination. All three fluorinated poly(3-alkyl)thiophenes displayed improved charge carrier mobilities despite a lower degree of crystallinity (as compared to P3HT), which may result from increased coplanarity, as indicated by conformational analysis using density functional theory (DFT). Second, Coughlin and coworkers increased the solubility of fluorinated P3HT via a direct (hetero)arylation (DHAP) protocol. $^{15}$ This was performed by varying the initial ratio of two monomers: 2-bromo-3hexylthiophene and 2-bromo-4-fluoro-3-hexylthiophene. However, with an increase in fluorinated thiophene content from 25 to $100 \%$, regioregularity dropped systematically from $89 \%$ to $78 \%$. Hole mobility of approximately $2-3 \times 10^{-5} \mathrm{~cm}^{2} \mathrm{~V}^{-1} \mathrm{~s}^{-1}$ (as determined by the space charge limited current method) and power conversion efficiency (PCE) of OPV devices also decreased with increased fluorination. The phenomenon of reduced PCE with reduced regioregularity has been previously observed in non-fluorinated $\mathrm{P} 3 \mathrm{HT} .{ }^{16}$ In the DHAP reaction, the presence of an electron-withdrawing group adjacent to the desired $\mathrm{C}-\mathrm{H}$ bond increases reactivity, ${ }^{17}$ which may have an adverse effect on the random nature of such a fluorinated copolymer. In addition to this, the authors used DHAP conditions suitable for copolymers but 
necessarily optimal for the preparation of poly(3-alkyl)thiophenes. ${ }^{18}$ These factors rendered an assessment of the impact of the random incorporation of fluorinated thiophene units into a P3HT chain difficult.

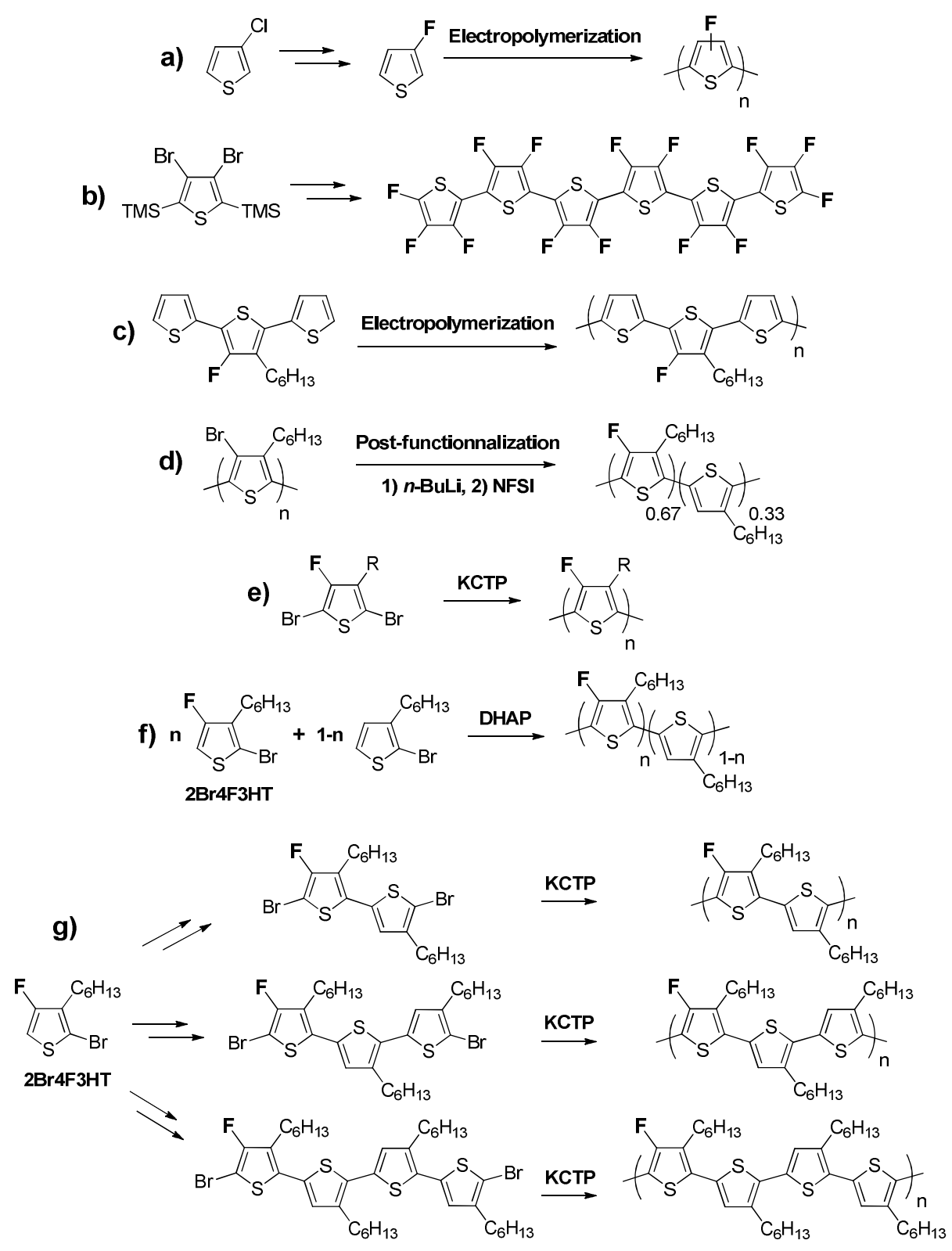

Figure 1. A summary of the synthesis of various fluorinated oligo- and polythiophenes from 1994 to the present: a) the electropolymerization of 3-fluorothiophene; ${ }^{9}$ b) the step-wise 
synthesis of tetradecafluorosexithiophene ${ }^{10}$ c) the electropolymerization of an alkylated monofluorinated terthiophene; ${ }^{12}$ d) the post-functionalization of brominated P3HT with the electrophilic fluorine source $N$-fluorobenzenesulfonimide (NFSI) ${ }^{13}$ e) the KCTP of 2,5-dibromo3-fluoro-4-alkylthiophenes; ${ }^{14}$ f) the random DHAP copolymerization of 2-bromo-4-fluoro-3hexylthophene with 2-bromo-3-hexylthiophene, $\left.{ }^{15} \mathrm{~g}\right)$ this report.

For this reason, we synthesized a series of highly regioregular fluorinated P3HT-based homopolymers via iterative thiophene couplings. We wished to assess the influence of fluorination on the optical and electronic properties of P3HT, especially with regards to the degree of fluorination required to modulate the HOMO and bandgap of P3HT. This experiment also served to determine if the increasing, precisely ordered presence of fluorine atoms along the polymer backbone leads to a change in charge-carrier mobility through electronic or steric influences. Kumada catalyst transfer polycondensation (KCTP) was used to allow more direct comparison with previously reported fully-fluorinated P3HT derivatives, which have been also prepared using this method. ${ }^{14}$

\section{Results and Discussion}

\section{Monomer Synthesis}

The starting fluorinated unit 2-bromo-4-fluoro-3-hexylthiophene (2Br4F3HT) was synthesized according to previously reported methods, ${ }^{10-12}$ albeit with a slight modification to the protocol of the fluorination step (see Supporting Information). The bromine-fluorine halogen exchange 
was undertaken with both the organolithium reaction mixture and the solution of freshly recrystallized $N$-fluorobenzenesulfonimide (NFSI) at $-100{ }^{\circ} \mathrm{C}$. This differs from previously recorded protocols, in which the reaction mixture was cooled to $-78{ }^{\circ} \mathrm{C}$ and the solution of NFSI was maintained at room temperature. The NFSI used was also freshly recrystallized in diethyl ether, according to a previously reported protocol. ${ }^{10}$ In this way, less dehalogenation was observed at the 3-position of the thiophene, and the isolated yield for this step was consequently improved from approximately 50 to $65 \%$.

A series of iterative stannylation reactions and Migita-Stille couplings was then undertaken in order to obtain compounds 4, 6 and $\mathbf{8}$ (as shown in Figure 2), in a similar fashion to a previous report. ${ }^{19}$ In each case, the 2-position of the thiophene was protected with a trimethylsilyl moiety in order to prevent stannylation at this position. An initial synthetic approach using $n$ butyllithium with tetramethylethylenediamine (TMEDA) was attempted in order to selectively insert the organotin moiety in the 5-position. As noted by Smith and Barratt, ${ }^{20}$ the use of amine ligands or even sterically hindered lithium amide reagents such as LiTMP (lithium 2,2,6,6tetramethylpiperidide) do not entirely eliminate the possibility of a mixture of regioisomers following electrophilic substitution. Even with high ratios of 5-substituted to 2-substituted thiophene derivatives, the presence of irregularly stannylated thiophenes result in some undesired coupling products, rendering purification procedures more difficult. 


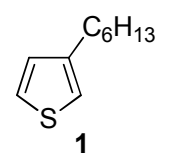

Following the preparation of the stannylated mono-, bi- and terthiophenes, each of these was coupled to 2Br4F3HT via Migita-Stille coupling, as presented in Figure 3. The desired products 9, 11 and $\mathbf{1 3}$ were obtained in $88 \%, 84 \%$ and $95 \%$ yield, respectively, and in each case the principal side-product was isolated and identified by ${ }^{1} \mathrm{H}$ NMR analysis as being the result of stannylthiophene homocoupling. Once the fluorinated thiophene unit was inserted onto the thiophene chain, the monomers were readily purified using reverse-phase (C-18) flash column chromatography, which turned out to be quite advantageous, as some impurities could not be 
separated from the desired product using silica as the stationary phase. This ensured the purity of the monomers and, by extension, the purity of the resulting polymers. Interestingly, the final step to obtain the di-brominated monomers 10, 12 and 14 required three different bromination reaction conditions (see Figure 3 for details). The bromination of 9 was performed as previously reported in numerous studies, by using N-bromosuccinimide (NBS) in a 1:1 mixture of chloroform and acetic acid, affording $\mathbf{1 0}$ in a 93\% yield. However, these conditions led to unexpected products when applied to $\mathbf{1 1}$ and 13. Following optimization of the bromination reaction, we found that the bromination of $\mathbf{1 1}$ with NBS afforded the desired product in acid-free chloroform. Acid-free chloroform was obtained by filtering the solvent through aluminum oxide (basic). In this way, 12 was obtained in 52\% yield. NBS was found to be an ineffective brominating agent in the preparation of 14, and 1,3-dibromo-5,5-dimethylhydantoin was employed to afford the desired product, albeit in an isolated yield of $51 \%$. All synthetic protocols are detailed in the Supporting Information.

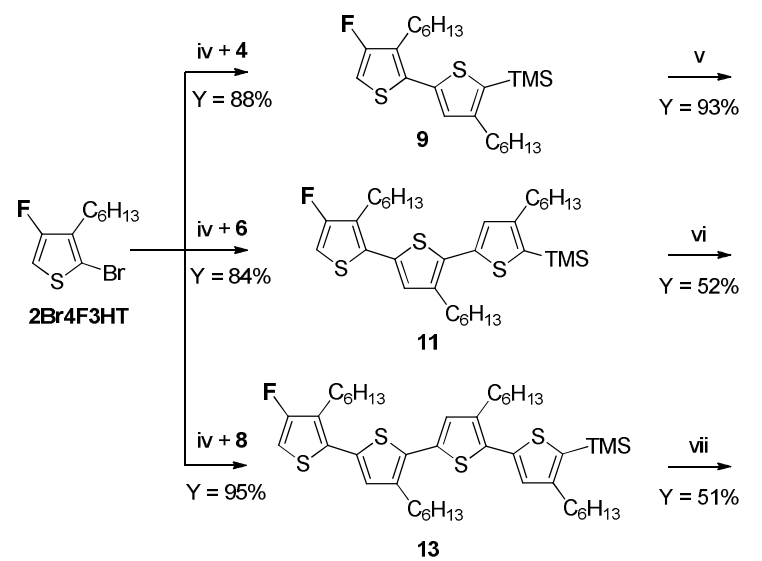

13

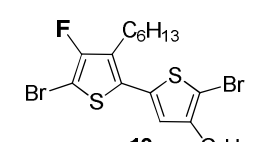

$10 \quad \mathrm{C}_{6} \mathrm{H}_{13}$

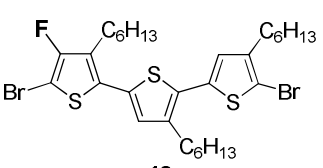

12
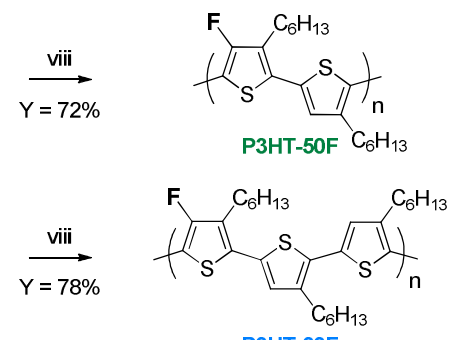

P3HT-33F

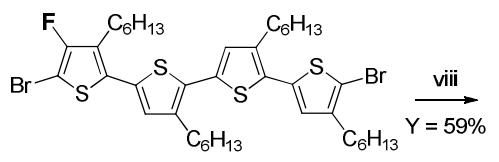

14

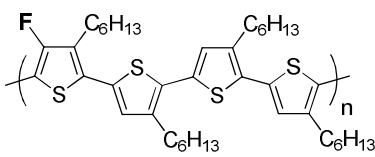

P3HT-25F

Figure 3. Synthesis of monomers from 2Br4F3HT coupled with stannyl thiophenes 4, 6 and 8 ; polymerization of 10, 12 and 14 to afford P3HT-50F, P3HT-33F and P3HT-25F, respectively. 
Reaction conditions: iv) $\mathrm{Pd}_{2} \mathrm{dba}_{3} 3 \mathrm{~mol} \%, \mathrm{P}(o \text {-tol })_{3} 12 \mathrm{~mol} \%$, toluene, reflux; v) NBS, $1: 1$ $\mathrm{CHCl}_{3}: \mathrm{AcOH}$; vi) $\mathrm{NBS}, \mathrm{CHCl}_{3}$; vii) 1,3-dibromo-5,5-dimethylhydantoin, $\mathrm{CHCl}_{3}$; viii) 0.97 $\mathrm{mol} \% \mathrm{i}$-PrMgCl$\cdot \mathrm{LiCl}, \mathrm{THF}, 0{ }^{\circ} \mathrm{C}$, then $\mathrm{Ni}(\mathrm{dppp}) \mathrm{Cl}_{2} 1.5 \mathrm{~mol} \%, 80^{\circ} \mathrm{C}$.

\section{Polymer Synthesis and Structural Characterization}

Polymerization of 10, 12 and 14, which afforded respectively P3HT-50F, P3HT-33F and P3HT-25F, was performed using similar conditions to those reported previously for P3HT and fluorinated P3HT. ${ }^{14,21-23}$ These consisted of selectively forming the Grignard on the fluorinated thiophene using the Turbo Grignard reagent $(i-\mathrm{PrMgCl} \cdot \mathrm{LiCl})$ in slightly sub-stoechiometric quantities (0.97 equivalent) at $0{ }^{\circ} \mathrm{C}$ in $\mathrm{THF}$, followed by the addition of the precatalyst $[1,3$ bis(diphenylphosphino)propane]dichloronickel(II) $\left(\mathrm{Ni}(\mathrm{dppp}) \mathrm{Cl}_{2}\right)$ to the reaction mixture and heating to $80^{\circ} \mathrm{C}$. Results for the three polymers are summarized in Table 1, along with those of P3HT prepared by both KCTP and DHAP. ${ }^{24}$

Table 1. Molecular weight, molar-mass dispersity, degree of polymerization, yield and regioregularity of P3HT-50F, P3HT-33F and P3HT-25F

\begin{tabular}{|c|c|c|c|c|c|}
\hline Polymer & $\bar{M}_{\boldsymbol{n}} \mathbf{( k g / m o l )}^{\boldsymbol{a}}$ & $\mathbf{D}_{\mathbf{M}}{ }^{\boldsymbol{a}}$ & $\mathbf{D P}^{\boldsymbol{b}}$ & Yield (\%) $^{\boldsymbol{c}}$ & RR (\%) $^{\boldsymbol{d}}$ \\
\hline P3HT-50F & 34 & 1.5 & 97 & 72 & 98.5 \\
\hline P3HT-33F & 46 & 1.3 & 88 & 78 & 98 \\
\hline P3HT-25F $^{\text {P3HT (KCTP) }}{ }^{e}$ & 53 & 1.5 & 78 & 59 & 95 \\
\hline P3HT (DHAP) $^{e}$ & 33 & 1.5 & 530 & - & 98 \\
\hline P3HT $^{e}$ & 1.8 & 199 & 96 & $>99$ \\
\hline
\end{tabular}


${ }^{a}$ Number average molar mass $\left(\bar{M}_{n}\right)$ and molar-mass dispersity $\left(\bigoplus_{\mathrm{M}}\right)$ were obtained from size exclusion chromatography (SEC) measurements. ${ }^{b}$ Degree of polymerization (DP) was calculated from the number average molar mass using the molar weight of the repeating unit. ${ }^{c}$ Yield was obtained following Soxhlet extraction and polymer washing. ${ }^{d}$ Regioregularity was determined from integration of the resonances in ${ }^{19} \mathrm{~F}$ NMR spectra. ${ }^{e}$ Previously-reported samples of P3HT. ${ }^{24}$

Crude polymers were precipitated in methanol and purified using Soxhlet extraction (acetone, hexane and ortho-dichlorobenzene $[o-\mathrm{DCB}]$ ), affording P3HT-50F, P3HT-33F and P3HT-25F with degrees of polymerization (DP) of 97, 88 and 78, and isolated yields of 72,78 and 59\%, respectively. Despite not being extracted with chloroform or chlorobenzene, molar-mass dispersity values remained relatively and comparatively narrow for each polymer. Some insoluble material remained in the Soxhlet cartridge following the extraction of P3HT-50F with $o$-DCB. The polymer washing step was used in order to reduce metallic impurities, following a reported procedure for polymers prepared by the KCTP method. ${ }^{25}$ P3HT-50F was found to be soluble in chlorinated solvents (o-DCB and 1,2,4-trichlorobenzene) above $100^{\circ} \mathrm{C}$, as was P3HT33F around $80^{\circ} \mathrm{C}$. P3HT-25F was found to be soluble in these solvents at room temperature.

MALDI-ToF analysis was undertaken in order to identify end-chains (full spectra are available in the Supporting Information). In every case, the signature peaks separated by the molecular weight of the repeating unit was well-defined. For all three polymers, the main peak could be attributed to $\mathrm{Br} / \mathrm{Br}$ end-chains and the secondary peak to the expected $\mathrm{H} / \mathrm{Br}$ end-chains. Interestingly, the loss of a $\mathrm{C}_{5} \mathrm{H}_{11}$ chain radical, which has been previously observed in $\mathrm{P}_{3} \mathrm{HT}^{26}$ 
and random fluorinated $\mathrm{P}_{3} \mathrm{HT}^{15}$ was not identified in any of the spectra. Nor was there any evidence of iso-propyl endgroups, potentially originating from brominated end groups reacting with unreacted iso-propylmagnesium chloride. Only in P3HT-25F were weak signals associated with $\mathrm{H} / \mathrm{H}$ end-chains found, possibly arising from magnesium-halogen exchange when the reaction mixture temporarily gelified at $80{ }^{\circ} \mathrm{C}$ (an increase in temperature to $120{ }^{\circ} \mathrm{C}$ was necessary to resolubilize the mixture) ${ }^{27}$ In $\mathrm{KCTP}, \mathrm{H} / \mathrm{Br}$ is the expected endcap pair, due to the quenching of the active Grignard chain ends with methanol. In this case, we hypothesized that the prevalence of $\mathrm{Br} / \mathrm{Br}$ endcapping may arise from reductive homocoupling of two Grignard chain ends (leading to the regeneration of the initial catalytic species $\mathrm{Ni}(\mathrm{dppp}) \mathrm{Cl}_{2}$ ), or from chain termination, in which the catalytic species simply decoordinates from the polymer chain before being inserted into a new $\mathrm{C}-\mathrm{Br}$ bond. This phenomenon becomes more likely as the reaction time increases. ${ }^{28}$ As described above, magnesium-halogen exchange could also be a cause of the observed end-chains, but as there is very little (P3HT-25F) or no evidence (P3HT-50F and P3HT-33F) of the accompanying $\mathrm{H} / \mathrm{H}$ originating from the quenching of the bis-Grignard polymer chain, this is unlikely. Due to the fact the molar masses observed in the MALDI-ToF spectra are considerably lower than those obtained from SEC and NMR measurements (see below), it can be presumed that only the shorter chains within the polymer sample are readily ionised when this method of characterization is used. This may lead to the disproportionately large fraction of $\mathrm{Br} / \mathrm{Br}$ end capped chains observed, as these were terminated early on in the polymerization reaction. It is therefore not possible to ascertain the nature of the end groups of longer polymer chains.

Polymer regioregularity and end-chains were studied by ${ }^{1} \mathrm{H}$ and ${ }^{19} \mathrm{~F}$ NMR analysis in tetrachloroethane (TCE) at $110^{\circ} \mathrm{C}$, due to the limited solubility of P3HT-50F and P3HT-33F at 
lower temperatures. The full ${ }^{1} \mathrm{H}$ NMR spectra as well as details of the aromatic and methylene regions are shown in Figure 4. The signal at $2.83 \mathrm{ppm}$ corresponds to the methylene of the fluorinated thiophene unit, and the signal at $2.91 \mathrm{ppm}$ to that of the non-fluorinated thiophene, although peak multiplicity was lost due to the heating necessary to fully solubilize P3HT-50F and P3HT-33F. The integration of these signals against one another serves as a tool to validate the polymer structure: the resonance at both 2.83 and $2.91 \mathrm{ppm}$ have the same integration value in P3HT-50F, the resonance at $2.91 \mathrm{ppm}$ is twice as intense as the resonance at $2.83 \mathrm{ppm}$ P3HT33F, and three time as intense in P3HT-25F. This effect can be readily correlated with the equivalent signals of the corresponding monomers: the well-defined triplet at ca. $2.57 \mathrm{ppm}$ corresponds to the methylene of fluorinated thiophene, and the multiplet centered on $\mathrm{ca} .2 .7 \mathrm{ppm}$ to those of all the other, non-fluorinated thiophenes. Coughlin and colleagues also observed this difference in methylene signals in their study of random fluorinated P3HT copolymers. ${ }^{15}$

In P3HT, the chemical shift of the central aromatic proton in the HT-HT triad is $6.98 \mathrm{ppm}$. In P3HT-50F, the principal aromatic resonance $\left(\mathrm{H}_{(\mathrm{A})}\right)$ is found at $7.14 \mathrm{ppm}$, indicating that the 50\% fluorinated equivalent of P3HT in the spectral conditions used $\left(110{ }^{\circ} \mathrm{C}\right)$ is downshifted by approximately 0.16 ppm vis-à-vis $\mathrm{P} 3 \mathrm{HT}$. The combination of the electronic effect of the fluorine atoms along the polymer backbone and the use of a different solvent (TCE) at a high temperature modifies the absolute chemical shift of the protons, but should not change the relative shift between protons. For this reason, it is possible to associate the secondary resonance visible in the P3HT-50F spectrum at $6.96 \mathrm{ppm}$ to a resonance which might appear at ca. $6.80 \mathrm{ppm}$ in P3HT. This corresponds most closely to the proton on a HT-HT brominated terminal thiophene $\left(\mathrm{H}_{(\mathrm{B})}\right.$, which is situated at $6.82 \mathrm{ppm}$ in non-fluorinated P3HT), confirming the results of MALDI-ToF analysis. It appears in 3\% of the intensity of the principal HT-HT signal, meaning that if, as the 
MALDI-ToF results indicate, the vast majority of end-chains are $\mathrm{Br} / \mathrm{Br}$, this corresponds to a degree of polymerization (DP) of 67 . The fact that the DP as calculated using NMR spectra is greatly inferior to the DP as determined by SEC measurements (97) is widely documented in the characterization of conjugated polymers, including poly(thiophene)s. ${ }^{29}$ This is due to the discrepancy of the hydrodynamic radius of such polymers as compared to the radii of the poly(styrene) standards used for the calibration of SEC methods.

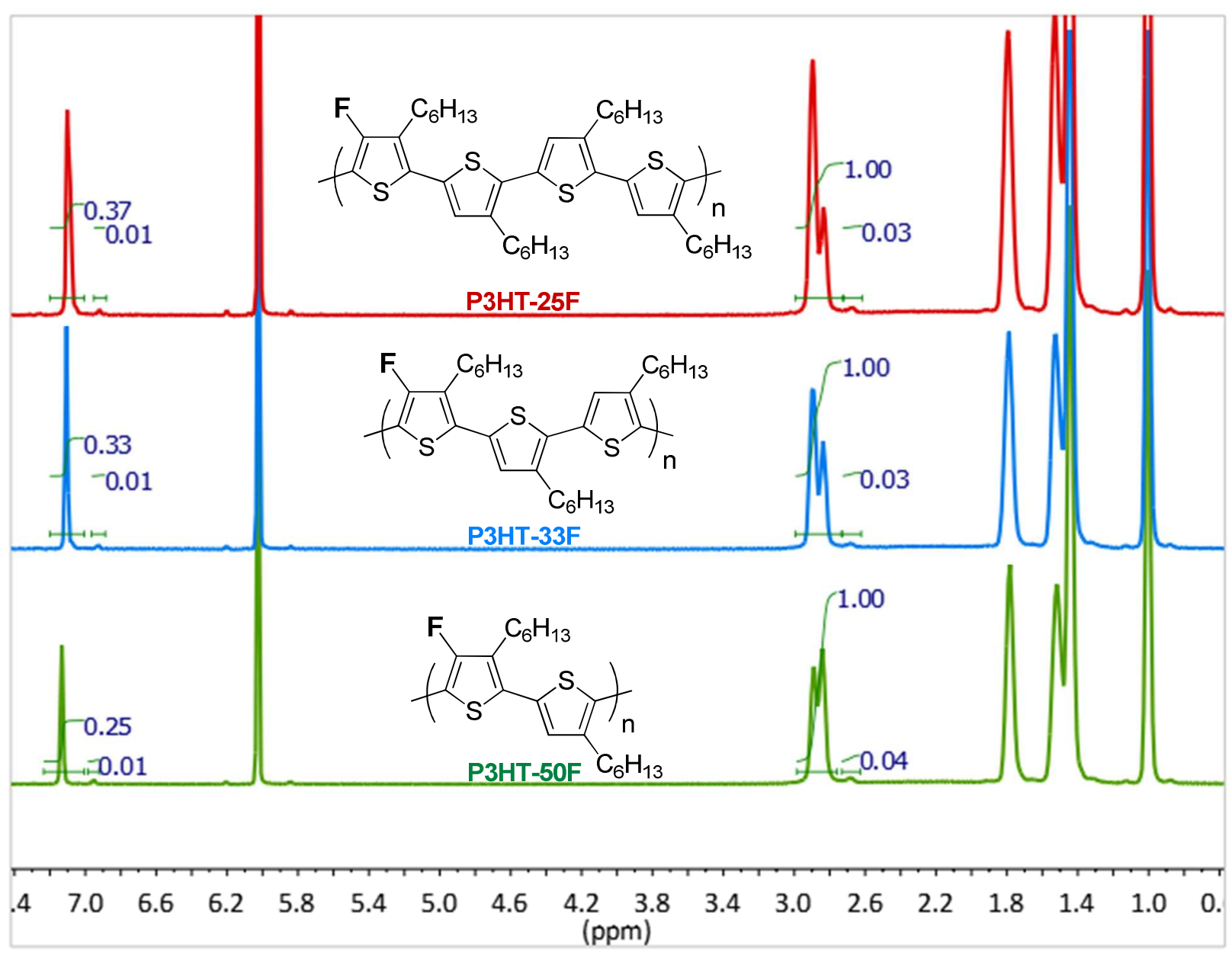




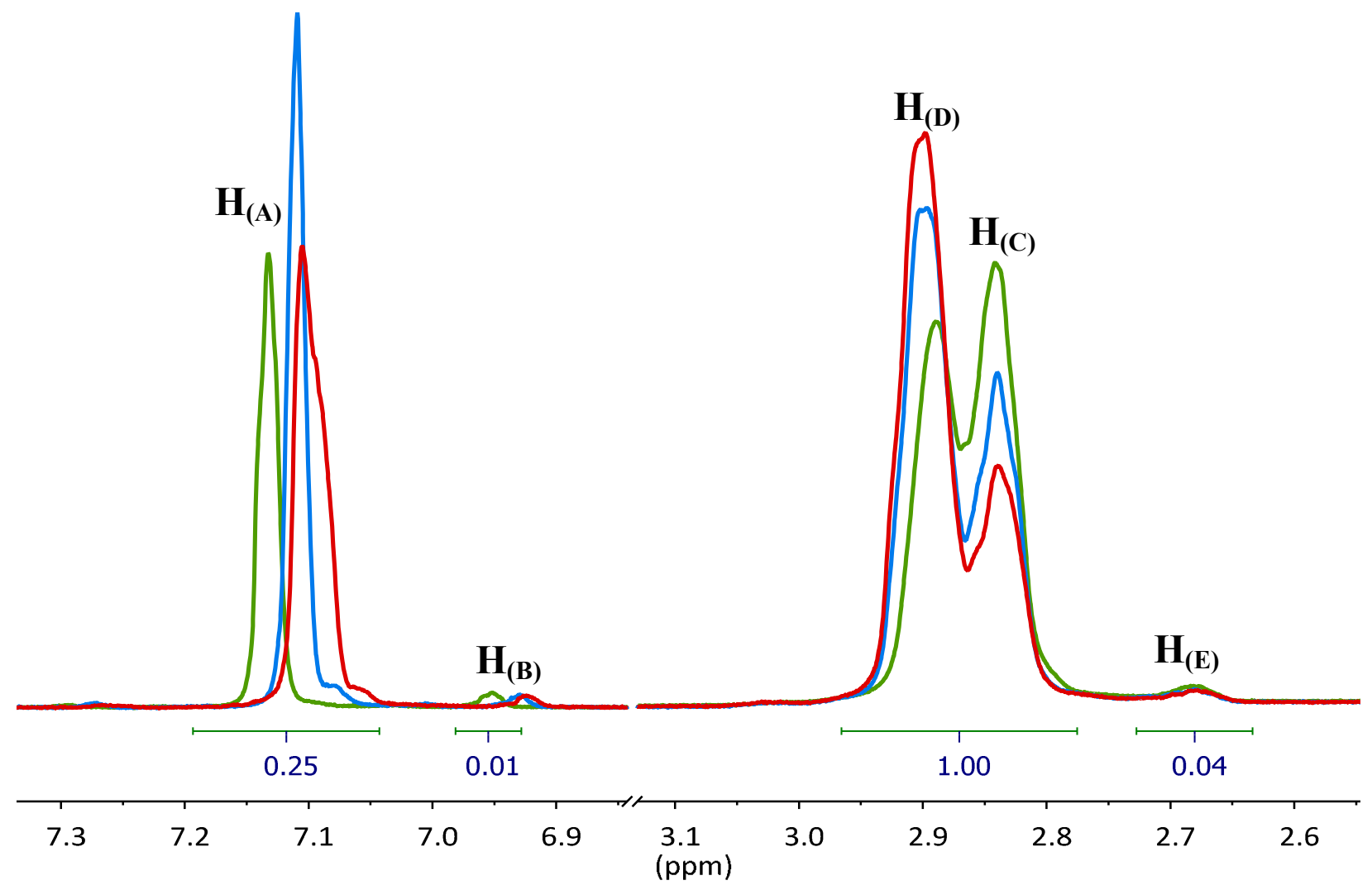

Figure 4. Full ${ }^{1} \mathrm{H}$ NMR spectra (above) and detail of the aromatic and methylene regions for P3HT-50F (green), P3HT-33F (blue) and P3HT-25F (red) in TCE at $110{ }^{\circ} \mathrm{C}$. The resonances at ca. $7.12 \mathrm{ppm}$ correspond to the protons on the non-fluorinated thiophenes $\left(\mathrm{H}_{(\mathrm{A})}\right)$. The resonances at $c a .2 .83$ ppm correspond to the first $\mathrm{CH}_{2}$ (methylene) of the hexyl chains of the fluorinated thiophene units $\left(\mathrm{H}_{(\mathrm{C})}\right)$, and those at ca. $2.91 \mathrm{ppm}$ to the methylene on non-fluorinated thiophenes $\left(\mathrm{H}_{(\mathrm{D})}\right) \cdot \mathrm{H}_{(\mathrm{B})}$ and $\mathrm{H}_{(\mathrm{E})}$ correspond to the aromatic and methylene protons on the end-chain units, respectively. Integration of P3HT-50F alone has been shown for clarity in the lower image. 
In addition to this, HT-HH defects in P3HT appear as a signal with a shift of $7.02 \mathrm{ppm}$. This would correspond to a resonance at 7.18 in P3HT-50F. There is no evidence of a resonance with this chemical shift, consistent with the fact that head-to-head homocoupling defects do not occur in P3HT prepared by the KCTP method. ${ }^{30}$ There is no other signal visible in the aromatic region of the ${ }^{1} \mathrm{H}$ NMR spectra, confirming both the polymer regioregularity and that initial TT coupling is the only observable defect within each polymer chain.

In the methylene region, a single secondary resonance appears at $2.69 \mathrm{ppm}$, upshifted by 0.20 ppm from the abovementioned principal methylene resonance of the non-fluorinated thiophene $\left(\mathrm{H}_{(\mathrm{D})}, 2.89 \mathrm{ppm}\right)$. The main explanation for this is an HT dyad end group. This is supported by the ${ }^{1} \mathrm{H}$ NMR spectrum of a sample of regioregular P3HT in the same analysis conditions used for P3HT-50F: although all resonance resolution is lost in these conditions, the secondary methylene resonance corresponding to the end-chains is also found at ca. $2.69 \mathrm{ppm}$, upshifted from the principal resonance by $0.21 \mathrm{ppm}$ (see Supporting Information for the P3HT spectrum). As the principal methylene resonances $\left(H_{(C)}\right.$ and $\left.H_{(D)}\right)$ occur in a $4: 1$ ratio to the principal aromatic resonance of P3HT-50F $\left(\mathrm{H}_{(\mathrm{A})}\right)$, so should the brominated chain-end methylene resonance appear in a 4:1 ratio to the aromatic proton on the brominated thiophene end group. This turns out to be the case, in that the secondary resonance at 2.69 ppm corresponds to $4 \%$ of the intensity of the two principal methylene resonances. This same reasoning was applied to the spectra of P3HT-33F and P3HT-25F, with similar results. The secondary resonances in the aromatic and methylene regions integrated for $3 \%$ of the principal resonances in both P3HT-33F and P3HT-25F.

In P3HT, the TT-HT triad produced by the initiation of KCTP chain growth can be observed in the aromatic region of ${ }^{1} \mathrm{H}$ NMR spectra. However, due to the presence of the fluorine atoms on 
the thiophene backbone in fluorinated P3HT derivatives, this is not possible. We therefore turned to interpreting the ${ }^{19} \mathrm{~F}$ NMR spectra of the three polymers for insight into the TT defect. Analyses reveal an upfield shift of the principal resonance $\left(F_{(A)}\right)$ going from $\delta=-120.97$ ppm in P3HT-50F to $\delta=-122.43 \mathrm{ppm}$ in P3HT-33F, and another slight shift to $\delta=-122.50 \mathrm{ppm}$ for P3HT-25F. In all of these spectra, a secondary signal upfield from the principal resonance is visible, and as the rate of fluorination decreases, this resonance draws nearer to the principal resonance. In P3HT-50F, this resonance has 3\% of the intensity of the principal resonance, and in P3HT-33F 4\%. In P3HT-25F the primary and secondary signals are differentiated from one another with difficulty, but the secondary resonance shows an intensity of approximately $10 \%$ relative to the principal resonance (see Supporting Information for full ${ }^{1} \mathrm{H}$ and ${ }^{19} \mathrm{~F}$ NMR spectra).

Due to the symmetrical nature of the inherent TT defect, the presence of fluorine atoms in each of the repeating units amplifies the defect twofold. If there is only one TT defect within the polymer chain, the 3\% intensity signal in P3HT-50F would therefore represent a TT defect $\left(\mathrm{F}_{(\mathrm{B})}\right)$ in approximately $1.5 \%$ of the polymer chain, hence a $98.5 \%$ regioregularity. This same reasoning leads to a regioregularity of 98\% for P3HT-33F and 95\% for P3HT-25F.

In the ${ }^{19} \mathrm{~F}$ NMR spectrum of P3HT-50F, a very weak signal can be observed at $-120.27 \mathrm{ppm}$. This may be produced by the small rate of TT initiation-induced homocoupling defects which, rather than occurring somewhere within the polymer chain, appear at the beginning of the chain, as was originally assumed to be the only position of the defect in KCTP-prepared polymers. One possible piece of evidence that this may be the case is that this resonance does not appear in the two other polymers. This may be due to the nature of the monomers used in that, as the rate of fluorination decreases, the fluorine atom is increasingly distant from the chain end, i.e. one 
thiophene separates it from the terminal C-Br bond in P3HT-50F, two in P3HT-33F and three in P3HT-25F. Therefore, the occurrence of a TT homocoupling defect present at a chain end for P3HT-33F and P3HT-25F would be too distant from the nearest fluorine atom for the electronic environment of P3HT-25F to be significantly different than a TT defect within the chain.

The values of regioregularity obtained using this method offer more of an indication as to the degree of polymerization for each chain, rather than the rate of defects. Since no other clear signals indicating other defects are present, one may conclude that there is only one TT defect per polymer chain. This TT defect is then 'diluted' in the number of regioregular HT couplings corresponding to the degree of polymerization of the chain. Despite the imprecision of NMR analysis in giving precise ratios for high molecular weight polymers, the values of regioregularity are in relatively good agreement with those of the soluble $100 \%$ fluorinated poly(3-alkylthiophene)s synthesized by Heeney et al. (in which the hexyl side chain was replaced by octyl and ethylhexyl chains). ${ }^{14}$

In P3HT, DSC measurements serve as an indicator of the degree of regioregularity. A study in which defects were deliberately incorporated into the polymer chain showed a strong correlation between the regioregularity and the crystallization temperature and enthalpy. ${ }^{31}$ Heating and cooling traces are shown in Figure 5, and Table $\mathbf{2}$ details the numerical values from these experiments, along with those of P3HT. All three samples display relatively sharp crystallization and melting peaks, indicating a high regioregularity for P3HT-50F, P3HT-33F and P3HT-25F. No evidence of glass transitions was found. With decreasing fluorination, the melting temperatures were 264,254 and $250^{\circ} \mathrm{C}$, crystallization temperatures were 239,228 and $221{ }^{\circ} \mathrm{C}$, and crystallization enthalpies were 32,27 and $25 \mathrm{~J} / \mathrm{g}$. The lower melting temperature and crystallization enthalpies for the polymers with a lower degree of fluorination may be due to 
reduced interactions between polymer chains. Melting and crystallization temperatures for P3HT-50F were only slightly lower than those reported for the 100\% fluorinated P3HT prepared by the same $\mathrm{KCTP}$ method $\left(\mathrm{T}_{\text {fus, }}=267{ }^{\circ} \mathrm{C}\right.$ and $\left.279{ }^{\circ} \mathrm{C} ; \mathrm{T}_{\mathrm{c}, \mathrm{p}}=245^{\circ} \mathrm{C}\right)$, and unlike the $100 \%$ fluorinated poly(3-alkylthiophene)s previously reported, P3HT-50F possessed a single melting peak, rather than two. ${ }^{14}$ Interestingly, the crystallization enthalpy of P3HT-50F was equivalent to that of the all-fluorinated P3HT. All three polymers also display high thermal stability, with nearly identical degradation temperatures.

Table 2. Physical properties of P3HT-50F, P3HT-33F, P3HT-25F, and P3HT

\begin{tabular}{|c|c|c|c|c|}
\hline Polymer & $\mathrm{T}_{\text {fus,p }}\left({ }^{\circ} \mathrm{C}\right)^{a}$ & $\mathrm{~T}_{\mathrm{c}, \mathrm{p}}\left({ }^{\circ} \mathrm{C}\right)^{a}$ & $\Delta H\left(\mathrm{~T}_{\mathrm{c}}\right)(\mathrm{J} / \mathrm{g})^{a}$ & $\mathbf{T}_{\mathrm{d}}\left({ }^{\circ} \mathrm{C}\right)^{b}$ \\
\hline P3HT-50F & 264 & 239 & 32 & 436 \\
\hline P3HT-33F & 254 & 228 & 27 & 438 \\
\hline P3HT-25F & 250 & 221 & 25 & 440 \\
\hline P3HT $\left(\right.$ KCTP) ${ }^{c}$ & 234 & 198 & 15 & 464 \\
\hline P3HT (DHAP) ${ }^{c}$ & 237 & 208 & 16 & 464 \\
\hline
\end{tabular}

${ }^{a}$ Melting temperature $\left(\mathrm{T}_{\text {fus,p }}\right)$, crystallization temperature $\left(\mathrm{T}_{\mathrm{c}, \mathrm{p}}\right)$ and enthalpy of crystallization $\left(\Delta H\left(\mathrm{~T}_{\mathrm{c}}\right)\right)$ were determined by differential scanning calorimetry (DSC) under nitrogen at a scanning speed of $10^{\circ} \mathrm{C} / \mathrm{min} .{ }^{b}$ Degradation temperature $\left(\mathrm{T}_{\mathrm{d}}\right)$ was determined at $5 \%$ weight loss via thermogravimetric analysis (TGA). ${ }^{c}$ Previously reported samples of P3HT. ${ }^{24}$ 


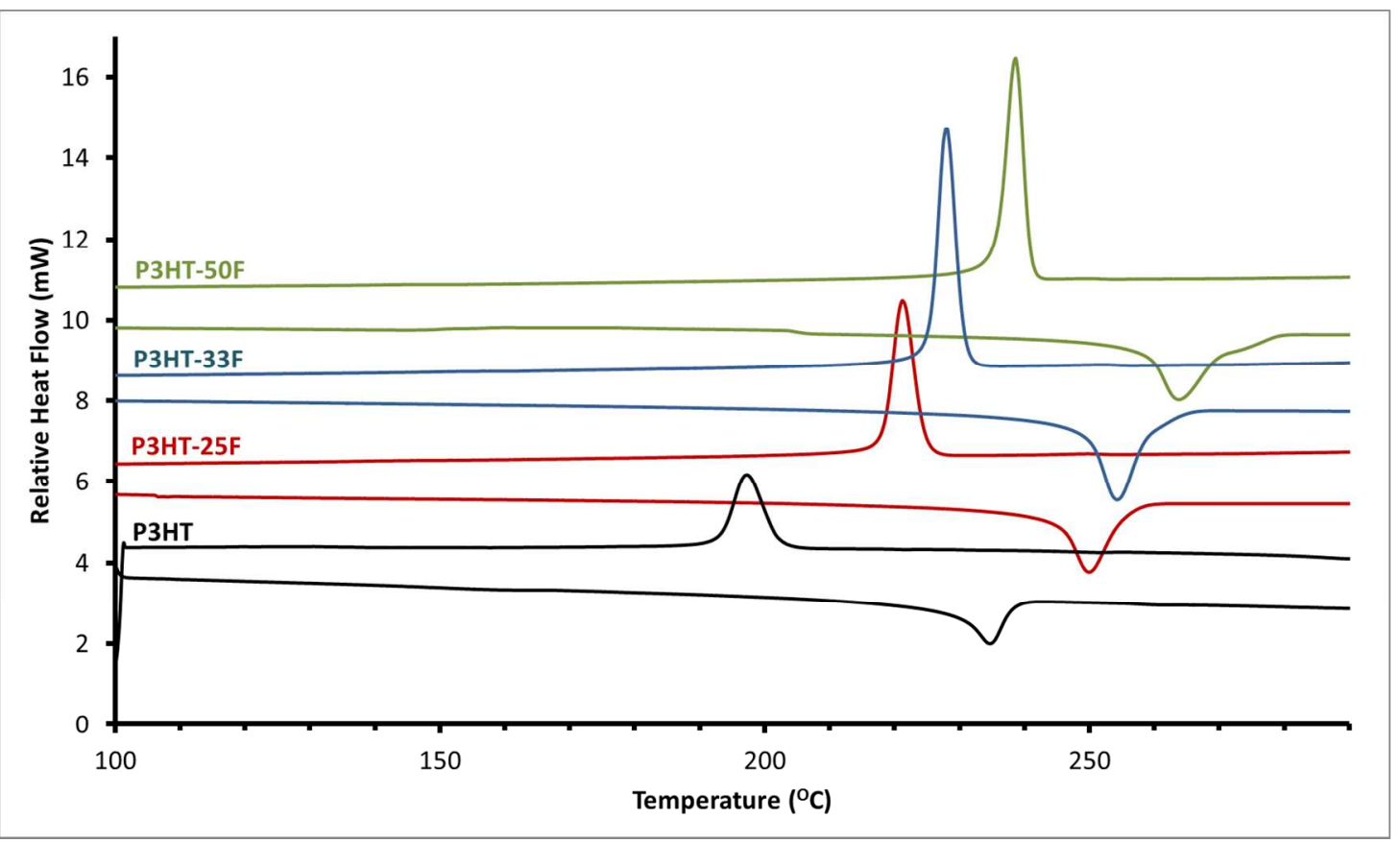

Figure 5. DSC traces for the second cycle of heating and cooling for P3HT-50F, P3HT-33F, P3HT-25F and P3HT under nitrogen at a scanning speed of $10{ }^{\circ} \mathrm{C} / \mathrm{min}$. Endothermic transitions point downward.

\section{Conformational analysis}

We employed density functional theory (DFT) calculations in order to understand the intrinsic conformational preferences of P3HT-50, P3HT-33 and P3HT-25. To reduce the computational expense and to simplify the potential energy surface, we truncated all alkyl sidechains to methyl groups with the common assumption that this modification would not substantially change their electronic properties. Our calculations were performed on model oligomers (monomers, dimers, and trimers) of the polymers studied experimentally (Figure 6). 


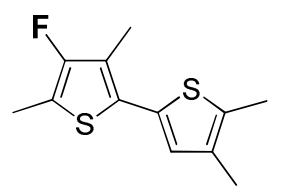

P3HT-50F-monomer

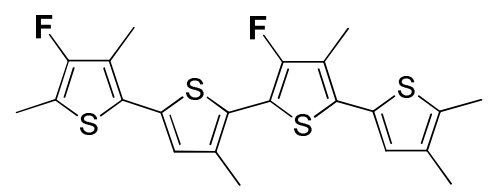

P3HT-50F-dimer

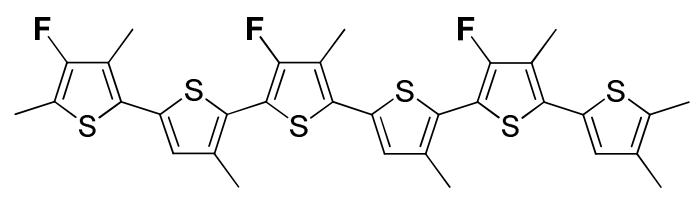

P3HT-50F-trimer

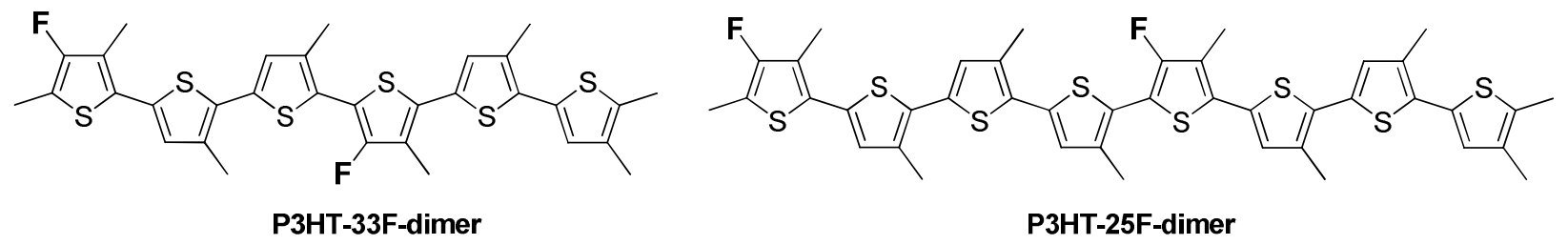

Figure 6. The series of truncated fluorinated P3HT oligomers studied using DFT calculations.

We employed the B3LYP/6-311+G(d,p)-D3BJ)-IEFPCM( $(\varepsilon=4) / / B 97 D / 6-31 G(d, p)-\operatorname{IEFPCM}(\varepsilon=4)$ level of theory to find the two lowest energy conformers based on this model chemistry for P3HT-50F-monomer. The structures and relative energies are shown in Figure 7.
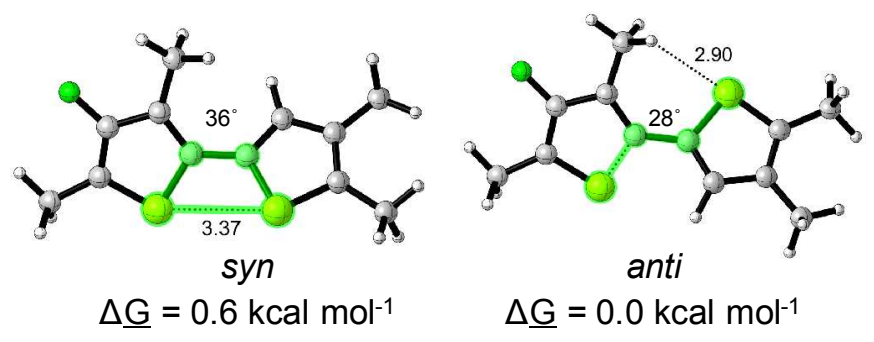

$\Delta \underline{G}=0.0 \mathrm{kcal} \mathrm{mol}^{-1}$

Figure 7. The lowest energy conformers of P3HT-50F-monomer. Computed using B3LYP/6$311+\mathrm{G}(\mathrm{d}, \mathrm{p})-\mathrm{D} 3 \mathrm{BJ})-\operatorname{IEFPCM}(\varepsilon=4) / / \mathrm{B} 97 \mathrm{D} / 6-31 \mathrm{G}(\mathrm{d}, \mathrm{p})-\operatorname{IEFPCM}(\varepsilon=4)$.

For P3HT-50F-monomer, the lowest energy conformer features the thiophenes pointing in opposite directions (anti). We refer to the dihedral angle between the two thiophenes as SCCS; the dihedral angle includes the sulfur atoms and connecting carbons between adjacent thiophenes. This conformation has a relatively distorted backbone; the SCCS dihedral angle is 
$28^{\circ}$. The structure is not planar in the gas phase because of competing S-S closed-shell repulsions and a steric clash between methyl hydrogens and the nearby sulfur lone pair orbitals (2.90 ̊). The syn conformer of P3HT-50F-monomer is higher in free energy by $0.6 \mathrm{kcal} \mathrm{mol}^{-1}$ and the backbone SCCS dihedral angle is $36^{\circ}$. The destabilizing S-S repulsion outweighs the $\mathrm{CH}_{3}-\mathrm{S}$ steric clash, and we expect this class of fluorinated thiophenes to exhibit more polymorphism and disorder than thiophenes unsubstituted at the 3-position. We extended our conformational search with P3HT-50F-dimer; Figure 8 shows the lowest energy conformer.
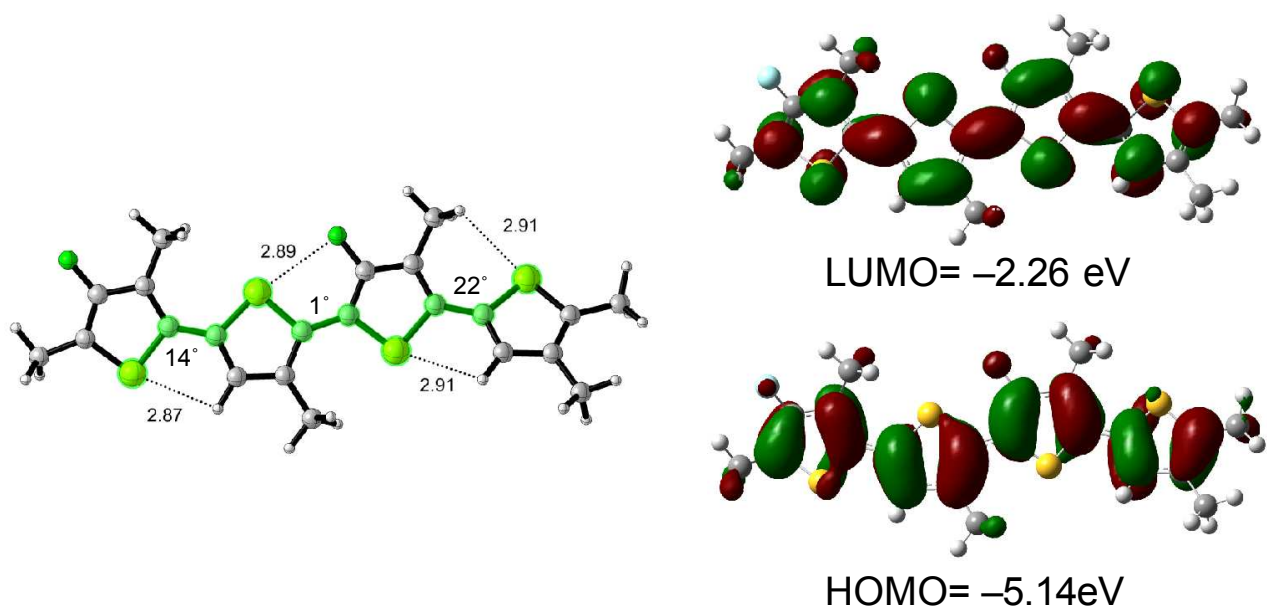

Figure 8. The lowest energy conformation of P3HT-50F-dimer. Computed using B3LYP/6$311+\mathrm{G}(\mathrm{d}, \mathrm{p})-\mathrm{D} 3 \mathrm{BJ})-\operatorname{IEFPCM}(\varepsilon=4) / / \mathrm{B} 97 \mathrm{D} / 6-31 \mathrm{G}(\mathrm{d}, \mathrm{p})-\operatorname{IEFPCM}(\varepsilon=4)$.

The lowest energy conformer features an all-anti relationship for the thiophene sulfurs. The central dihedral angle is virtually planar $\left(1^{\circ}\right.$ rotated from planarity). De la Cruz et al. have suggested that S-F interactions are slightly stabilizing, ${ }^{32}$ which appears to preserve planarity about the central SCCS dihedral in P3HT-50-dimer. The outermost thiophenes are more distorted from planarity $\left(14^{\circ}\right.$ and $\left.22^{\circ}\right)$ because of $\mathrm{CH}_{3}-\mathrm{S}$ steric clashes identified in Figure 8. We then computed P3HT-50-trimer, but only considered the all-syn and all-anti conformers. The 
all-syn conformer is higher in free energy by $1.2 \mathrm{kcal} \mathrm{mol}^{-1}$ and is helical in the gas phase to avoid three pairs of S-S repulsions. Only the all-anti conformation of P3HT-50-trimer is considered, and is shown in Figure 9.

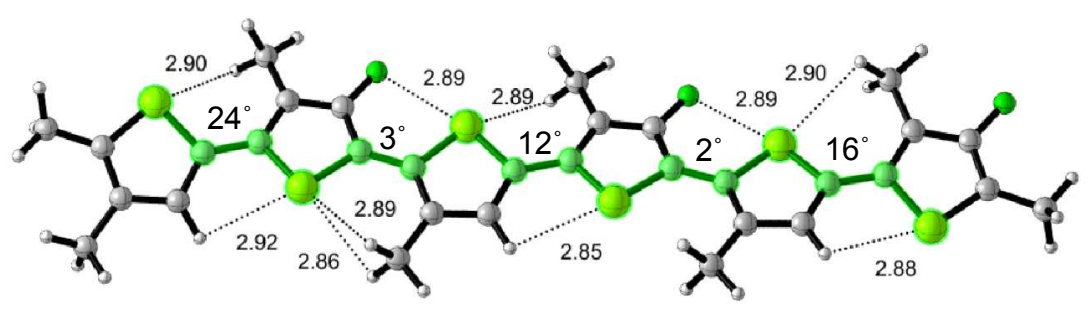

Figure 9. The all-anti conformation of P3HT-50-trimer, computed using B97D/6-31G(d,p)$\operatorname{IEFPCM}(\varepsilon=4)$.

Wherever S-F interactions are possible, the corresponding SCCS dihedral angles are considerably more planar $\left(2-3^{\circ}\right.$ vs $\left.12-24^{\circ}\right)$. Again, SCCS dihedral angles for the outermost thiophenes are larger $\left(16^{\circ}\right.$ and $\left.24^{\circ}\right)$ to avoid $\mathrm{CH}_{3}-\mathrm{S}$ steric repulsions. We report the computed HOMO and LUMO energies for these systems in Table 3. 
Table 3. The computed frontier molecular orbitals for $\mathbf{1}$ in the all-anti conformations.

\begin{tabular}{|c|c|c|c|}
\hline $\begin{array}{c}\text { Truncated oligomer } \\
\text { model }\end{array}$ & $\begin{array}{c}\text { HOMO } \\
\mathbf{( e V})\end{array}$ & $\begin{array}{c}\text { LUMO } \\
\mathbf{( e V})\end{array}$ & gOp (eV) \\
\hline P3HT-50-monomer & -5.69 & -1.34 & 4.35 \\
\hline P3HT-50-dimer & -5.14 & -2.26 & 2.88 \\
\hline P3HT-50-trimer & -4.99 & -2.51 & 2.48 \\
\hline P3HT-33-dimer & -4.91 & -2.42 & 2.49 \\
\hline P3HT-25-dimer & -4.82 & -2.53 & 2.29 \\
\hline
\end{tabular}

Table 3 shows that the HOMO-LUMO gap is extremely large for the monomer $(4.35 \mathrm{eV})$ because of the short conjugation length in this oversimplified system. The dimer and trimer approach those measured experimentally (Table 4, see below). Our results are consistent with many other reports of decreasing HOMO and LUMO energies with increased proportions of fluorinated backbone. The HOMO-LUMO gap is larger for P3HT-50-dimer than P3HT-33dimer and P3HT-25-dimer because fluorination lowers the energy of the P3HT-50F HOMO more than that of the LUMO. This behavior is due to the fluorine lone pair being part of the HOMO, but not the LUMO.

For P3HT-33 and P3HT-25, we computed the truncated structures P3HT-33-dimer and P3HT25-dimer in the all-anti conformation and calculated the corresponding frontier molecular orbitals (Figure 10). 

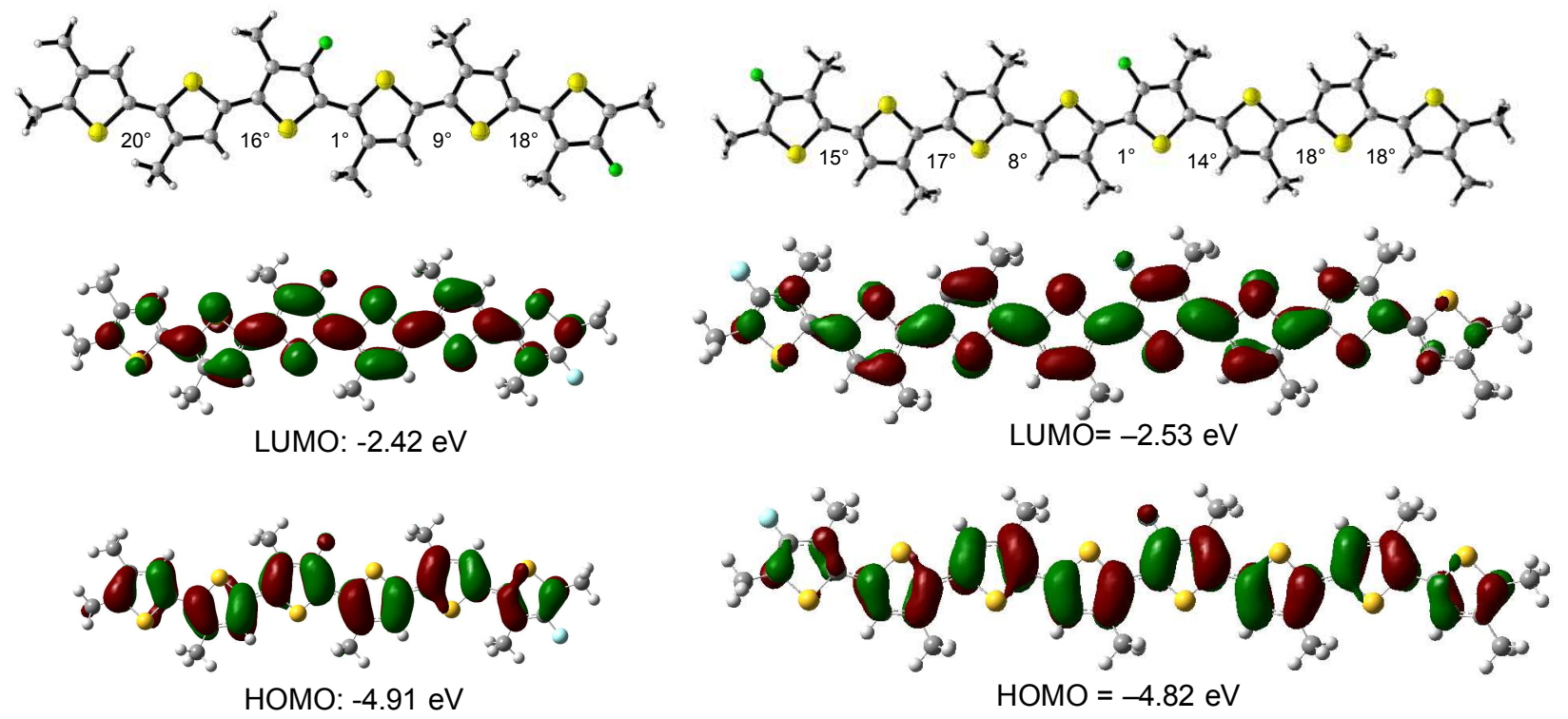

Figure 10. The structure of P3HT-33-dimer and P3HT-25-dimer with the corresponding frontier molecular orbitals. Computed using B3LYP/6-311+G(d,p)-D3BJ)$\operatorname{IEFPCM}(\varepsilon=4) / / \mathrm{B} 97 \mathrm{D} / 6-31 \mathrm{G}(\mathrm{d}, \mathrm{p})-\operatorname{IEFPCM}(\varepsilon=4)$.

In P3HT-33-dimer, the SCCS dihedral angles range from $1-20^{\circ}$ and in P3HT-25-dimer the dihedral angles range from $1-18^{\circ}$, with the central SCCS dihedral angle virtually planar $\left(1^{\circ}\right.$ out of plane twist). Through-space S-F interactions reinforce the planarity and $\mathrm{CH}_{3}-\mathrm{S}$ steric clashes increase the SCCS angles in these dimers. The extensive delocalization of the frontier molecular orbitals of P3HT-25-dimer relative to P3HT-33-dimer results in a smaller HOMO-LUMO gap (2.29 vs. $2.49 \mathrm{eV})$. 


\section{Polymer Optical and Electronic Properties}

Solution UV-visible analyses were undertaken using a temperature gradient in order to assess the aggregation of the polymers in solution (solution and solid-state UV-visible spectra are shown in Figure 11, and numerical values from solution and film UV-visible spectroscopic analyses are detailed in Table 4). The spectra of fluorinated polymers are highly temperature-dependent, as previously reported. ${ }^{33}$ Results indicate that P3HT-25F is soluble in $o$-DCB at room temperature and that P3HT-33F becomes fully solubilized at ca. $70^{\circ} \mathrm{C}$. In P3HT-50F, the shape of the absorbance spectrum indicates the presence of aggregates, which persist until $100^{\circ} \mathrm{C}$. Above these temperatures, all three polymers produce similar, featureless spectra with absorbance maxima at 422, 430 and $432 \mathrm{~nm}$ for P3HT-50F, P3HT-33F and P3HT-25F, respectively. The temperature-dependant evolution of the spectra was found to be reversible.

Following the observation of these aggregates at relatively high temperatures, films for solidstate absorbance spectra were spin-coated from $o$-DCB solutions at $120^{\circ} \mathrm{C}$ in order to ensure the full solubility of all three polymers prior to deposition. The three polymers display almost identical absorbance spectra in the solid state, indicating similar inter- and intra-chain organization upon cooling. The two principal absorbance maxima appear at 548/596, 550/595 and 553/600 nm for P3HT-50F, P3HT-33F and P3HT-25F, respectively, which can be attributed to the $\mathrm{A}_{0-0}$ and $\mathrm{A}_{0-1}$ spectral transitions. ${ }^{34}$ The slight variation in absorbance is not due to variation in molecular weights, as all three polymers have surpassed the effective conjugation length of poly(thiophene)s at the observed chain length. ${ }^{35}$ With decreased fluorination, both spectral transitions shift towards longer wavelengths and approach the standard values of P3HT in the solid state $(555 / 610 \mathrm{~nm}) .^{36}$ 
The ratio of the intensity of these two absorbance maxima $\left(\mathrm{A}_{0-0} / \mathrm{A}_{0-1}\right)$ is comparable for the three polymers. In $\mathrm{P} 3 \mathrm{HT}$, a high $\mathrm{A}_{0-0} / \mathrm{A}_{0-1}$ ratio is indicative of both a high regioregularity and high degree of polymerization. A substantial increase of DP would be necessary to compensate for the impact of a small reduction in regioregularity on the $\mathrm{A}_{0-0} / \mathrm{A}_{0-1}$ ratio, due to the effective conjugation length in the crystallized polymer. ${ }^{34}$ Consequently, the similar $\mathrm{A}_{0-0} / \mathrm{A}_{0-1}$ ratios of the three polymers $(0.965,0.920$ and 0.956 , respectively) fits with the slight reduction in regioregularity from P3HT-50F to P3HT-25F (98\%, 97\% and 95\%) at the same time as a relatively large increase of molecular weight values. On the other hand, as observed from NMR spectra, the only noticeable defect is the tail-to-tail homocoupling issuing from the initiation of the KCTP reaction, as discussed earlier. Unlike a head-to-head defect, a tail-to-tail defect in the polymer would likely not induce twisting of the conjugated backbone. It is possible that the presence of fluorine substituents in adjacent thiophene units may in fact favor localized coplanarity and rigidity. Therefore, the regioregularity may have a negligible effect on the observed $\mathrm{A}_{0-0} / \mathrm{A}_{0-1}$ values.

The optical bandgap, as calculated using the solid-state UV-visible spectra, changes very little between the three polymers, indicating that both the HOMO and LUMO levels deepen in parallel with an increased rate of fluorination (numerical values of electronic measurements are given in Table 4). However, the observed bandgaps are slightly larger than reported values for P3HT (1.9 eV). ${ }^{37}$ The bandgap is very similar to the previously reported randomly fluorinated P3HT analogues, although the bandgap increases from 1.93 in the $25 \%$ fluorinated polymer to $1.99 \mathrm{eV}$ in the $100 \%$ fluorinated polymer. ${ }^{15}$ The similar bandgaps for P3HT-50F, P3HT-33F and P3HT25F may be indicative of the comparable regioregularity of the three polymers. 
Table 4. Optical and electronic measurements for P3HT-50F, P3HT-33F and P3HT-25F

\begin{tabular}{|c|c|c|c|c|c|c|c|}
\hline Polymer & $\begin{array}{l}\mathbf{A}_{0-0} \\
/ \mathbf{A}_{0-1}\end{array}$ & Film $^{a}$ & $\begin{array}{l}\text { Solution } \\
\text { (RT in } o- \\
\text { DCB })^{b}\end{array}$ & $\begin{array}{l}\text { Solution } \\
\left(140^{\circ} \mathrm{C} \text { in }\right. \\
o-\mathrm{DCB})^{b}\end{array}$ & $\begin{array}{l}\mathbf{E}_{\mathrm{g} \mathrm{opt}} \\
(\mathrm{eV})^{c}\end{array}$ & $\begin{array}{l}\text { HOMO } \\
(\mathrm{eV})^{d}\end{array}$ & $\begin{array}{c}\text { LUMO } \\
(\mathrm{eV})^{e}\end{array}$ \\
\hline P3HT-50F & 0.965 & 548,596 & 548,594 & 422 & 1.98 & -5.34 & -3.36 \\
\hline P3HT-33F & 0.920 & 550,595 & 460,594 & 430 & 1.98 & -5.26 & -3.28 \\
\hline P3HT-25F & 0.956 & 553,600 & 462 & 432 & 1.97 & -5.24 & -3.27 \\
\hline
\end{tabular}

${ }^{a}$ Solid-state UV-visible spectra were obtained from as-spun thin films prepared via spin coating of a $2.0 \mathrm{mg} / \mathrm{ml}$ solution of the polymer in $o-\mathrm{DCB}$ at $120{ }^{\circ} \mathrm{C}$. ${ }^{b}$ Solution UV-visible spectra were obtained from a $0.01 \mathrm{mg} / \mathrm{ml}$ solution of the polymer heated until fully soluble in $o$-DCB and then cooled to the desired temperature for analysis. ${ }^{c}$ Optical bandgap for each polymer was determined using the tangent of the onset of low-energy absorbance in the corresponding UVvisible spectrum. ${ }^{d}$ The onset of oxidation was determined by cyclic voltammetry of polymer films cast on the electrode from an $o$-DCB solution; an external reference of ferrocene $\left(\mathrm{Fc} / \mathrm{Fc}^{+}\right)$ was used. ${ }^{e}$ The LUMO energy was estimated by subtracting the optical bandgap $\left(\mathrm{E}_{\mathrm{g} \text { opt }}\right)$ from the observed HOMO. 

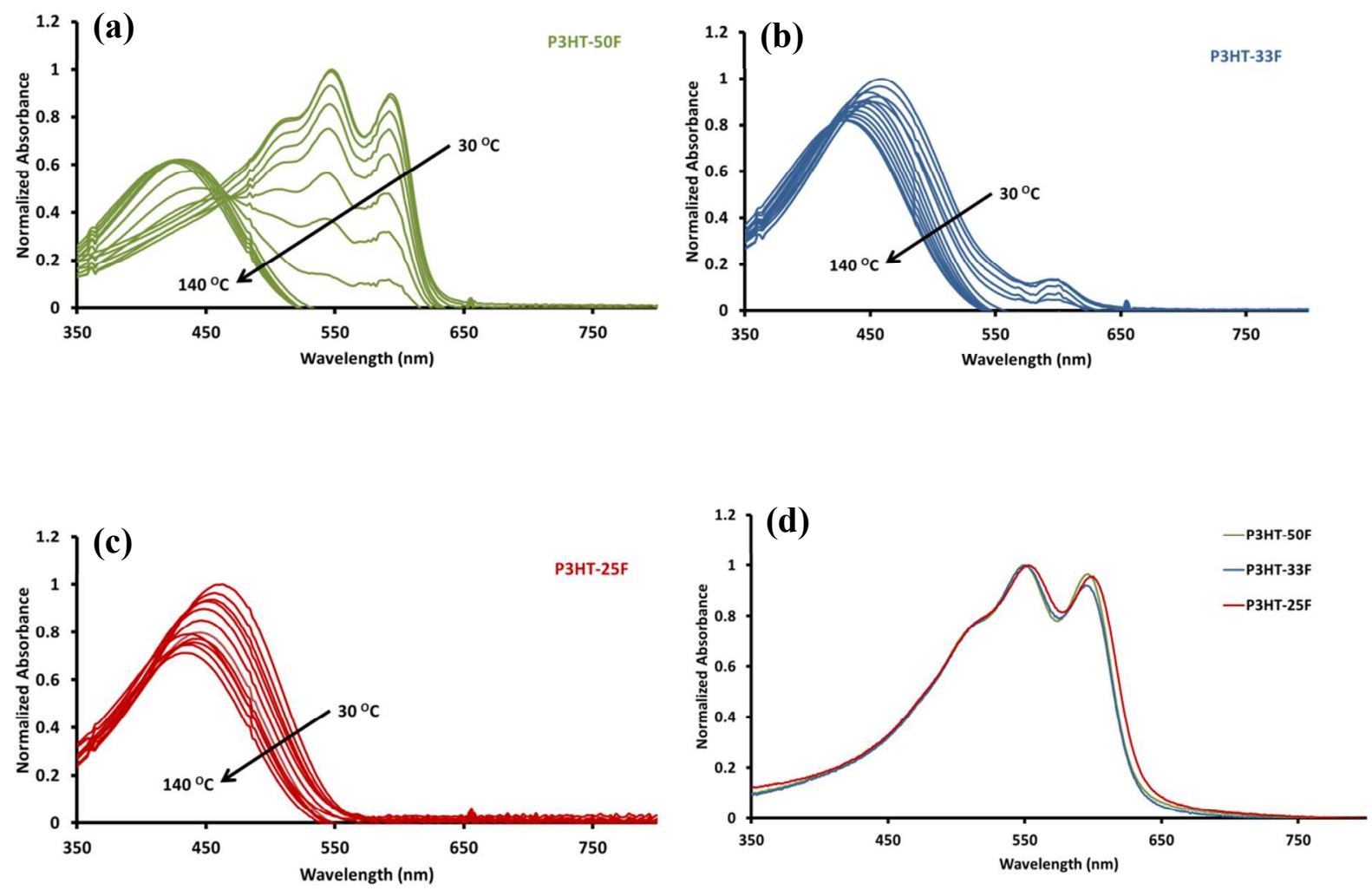

Figure 11. UV-visible spectra of polymers P3HT-50F, P3HT-33F and P3HT-25F in solution ( $a, b$ and $c$ ) and in thin films (d). Solid-state UV-visible spectra were obtained from as-spun thin films prepared via spin coating of a $2.0 \mathrm{mg} / \mathrm{ml}$ solution of the polymer in $o$-DCB at $120{ }^{\circ} \mathrm{C}$. Solution UV-visible spectra were obtained from a $0.01 \mathrm{mg} / \mathrm{ml}$ solution of the polymer heated until fully soluble in $o$-DCB and then cooled to $30^{\circ} \mathrm{C}$, at which point the temperature was increased in $10^{\circ}$ increments.

Cyclic voltammetry was used to compare the onset of oxidation of the three polymers with that of P3HT. Cyclic voltammograms for P3HT-50F, P3HT-33F and P3HT-25F, as well as for P3HT are shown in Figure 12, along with that of the external ferrocene reference (numerical 
values are found in Table 4). In all three voltammograms, three oxidative doping peaks are clearly visible. Due to this similarity with the voltammograms of P3HT, it is possible that these peaks also correspond to the formation of a polaron and bipolaron. The reductive peaks are also somewhat visible in all three polymers. P3HT-50F shows the most narrow oxidation peaks, perhaps due to the narrow spacing of the fluorinated thiophenes which may lead to the most isoenergetic polymer chain conjugation. ${ }^{38}$ The three oxidation peaks in P3HT-25F appear at a higher voltage than those of the two other fluorinated polymers and, in keeping with this, the onset of oxidation of P3HT-50F, P3HT-33F and P3HT-25F is -5.34, -5.26 and -5.24 eV, respectively, as compared to $-5.18 \mathrm{eV}$ for the $\mathrm{P} 3 \mathrm{HT}$ reference polymer analyzed in the same conditions. It may be concluded that below 33\%, fluorination has very little effect on the electrochemical properties of P3HT, as the oxidation onset between P3HT-33F and P3HT-25F changes very little. A parallel can be drawn here with the study of DHAP-prepared random fluorinated copolymers, the authors of which identified that the HOMO changes very little between the $50 \%, 75 \%$ and $100 \%$ fluorinated materials, possibly due to 'donor' and 'acceptor' effects. ${ }^{15}$ The electronic effect of the fluorine substituent on the HOMO does therefore seem to have the greatest impact somewhere between 33\% and 50\% substitution, and beyond this point increased fluorination only reduces solubility. 


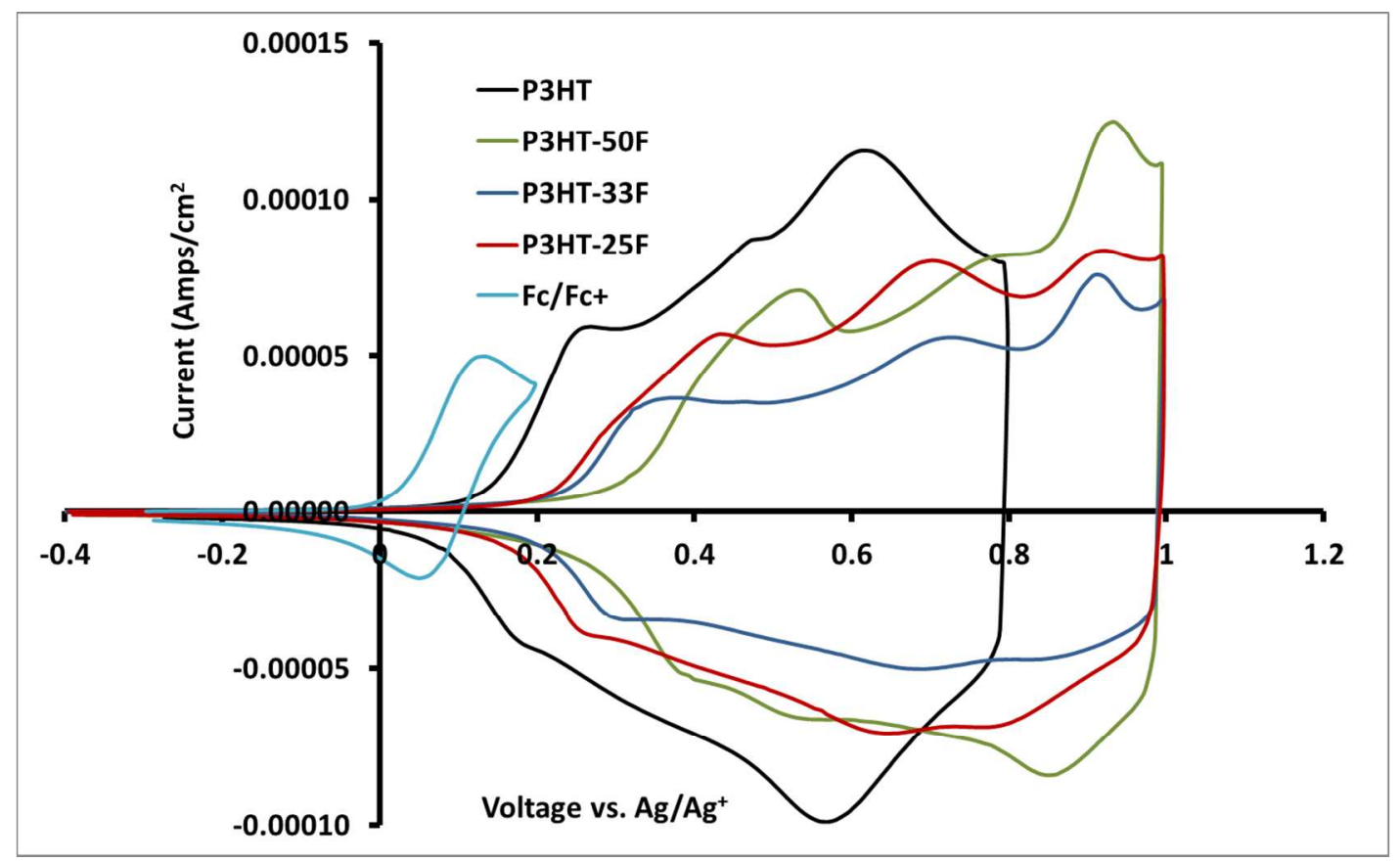

Figure 12. Cyclic voltammetry measurements for P3HT-50F, P3HT-33F and P3HT-25F, along with a P3HT sample and the external ferrocene $\left(\mathrm{Fc} / \mathrm{Fc}^{+}\right)$reference. Polymer films were cast onto the electrode from an $o$-DCB solution.

For comparison purposes, field effect transistors were prepared in the bottom-gate bottomcontact configuration (BGBC) to assess charge carrier mobilities in the three polymers. Due to the limited solubility of P3HT-50F, all polymers were deposited via spin-coating of an $o$-DCB solution. Full details of transistor preparation are available in the Supporting Information. Average and maximum charge carrier mobilities, on/off current ratios and threshold voltages for each polymer at these two temperatures are detailed in Table 5, and are accompanied by reference values for P3HT. The hole mobility of P3HT-50F $\left(0.0079 \mathrm{~cm}^{2} \mathrm{~V}^{-1} \mathrm{~s}^{-1}\right)$ and P3HT-25F 
$\left(0.0013 \mathrm{~cm}^{2} \mathrm{~V}^{-1} \mathrm{~s}^{-1}\right)$ were found to be optimal at $100{ }^{\circ} \mathrm{C}$ and the highest average was obtained with P3HT-33F at $150{ }^{\circ} \mathrm{C}\left(0.0289 \mathrm{~cm}^{2} \mathrm{~V}^{-1} \mathrm{~s}^{-1}\right)$, although mobility values were comparable at these two annealing temperatures. The fact that P3HT-33F led to the best results was possibly due to an optimized trade-off between the increasing planarity of the polymer backbone and decreasing crystallinity when the degree of fluorination is increased, as observed in previous fluorinated polythiophenes. ${ }^{14,} 15$ In similarly-prepared devices, highly regioregular nonfluorinated P3HT prepared by both KCTP and DHAP displays much higher mobilities (0.08 and $0.14 \mathrm{~cm}^{2} \mathrm{~V}^{-1} \mathrm{~s}^{-1}$, respectively). ${ }^{24} \mathrm{It}$ is, however, interesting to note that on/off current ratios for all three polymers surpass not only those of non-fluorinated P3HT which was prepared in the same conditions $\left(10^{3}\right)$, but also those of previously reported all-fluorinated P3HT $\left(10^{4}-10^{5}\right) .{ }^{14,24}$ The most likely explanation may be related to the deeper HOMO levels $(\sim-5.3 \mathrm{eV}$ as opposed to $\sim$ 5.2 for P3HT). Indeed, the three fluorinated polymers are less susceptible to doping by ambient oxygen than P3HT, preserving their insulating properties and, by extension, limiting their electrical conductivity and minimizing their off current. No electron transport behavior was observed for any of the three polymers. 
Table 5. Average and maximum mobility in field-effect transistors.

\begin{tabular}{|c|c|c|c|c|}
\hline Polymer & $\left.\mathbf{T ~} \mathbf{(}^{\mathbf{0}}\right)^{\boldsymbol{a}}$ & $\boldsymbol{\mu}\left(\times \mathbf{1 0}^{-\mathbf{2}} \mathbf{c m}^{\mathbf{2}} \mathbf{V}^{-\mathbf{1}} \mathbf{s}^{-\mathbf{1}}\right)^{\boldsymbol{b}}$ & $\mathbf{I}_{\mathrm{ON} / \mathrm{OFF}}{ }^{c}$ & $\mathbf{V}_{\mathbf{T H}}{ }^{d}$ \\
\hline \multirow{2}{*}{ P3HT-50F } & 100 & $0.45 \pm 0.20(0.79)$ & $\sim 10^{5}-10^{6}$ & 6.9 \\
\cline { 2 - 5 } & 150 & $0.43 \pm 0.20(0.75)$ & $\sim 10^{6}$ & 7.1 \\
\hline \multirow{2}{*}{ P3HT-33F } & 100 & $2.66 \pm 0.13(2.82)$ & $\sim 10^{6}$ & 10.5 \\
\cline { 2 - 5 } & 150 & $2.72 \pm 0.16(2.89)$ & $\sim 10^{6}-10^{7}$ & 7.9 \\
\hline \multirow{2}{*}{ P3HT-25F } & 100 & $1.18 \pm 0.07(1.30)$ & $\sim 10^{6}-10^{7}$ & 7.7 \\
\cline { 2 - 5 } & 150 & $1.05 \pm 0.07(1.17)$ & $\sim 10^{5}-10^{6}$ & 1.1 \\
\hline
\end{tabular}

${ }^{a}$ Annealing temperature. ${ }^{b}$ Charge carrier mobility values are written out in the form Average \pm standard deviation (maximum). ${ }^{c}$ On/off current ratio. ${ }^{d}$ Average threshold voltage.

\section{Conclusion}

Three derivatives of poly(3-hexylthiophene) which differ in the ratio of fluorinated thiophene repeating units were reported. P3HT-50F, P3HT-33F and P3HT-25F were synthesized via the Kumada catalyst transfer polymerization of monomers which were prepared in turn using iterative Migita-Stille couplings from 2-bromo-4-fluoro-3-hexylthiophene (2Br4F3HT). This method allowed for a regular separation of fluorine atoms along the polythiophenes backbone. Detailed ${ }^{1} \mathrm{H}$ and ${ }^{19} \mathrm{~F}$ NMR spectroscopic studies was undertaken, and from these it could be determined that all three polymers displayed comparable and high regioregularity. Well-defined 
UV-visible absorbance spectra and sharp peaks in differential scanning calorimetry measurements both confirmed the high regioregularity of the three polymers. Computational analyses indicated that favourable fluorine-sulfur interactions contribute to the planarity of the anti-conformation between thiophene units of truncated models. The dihedral angle between two thiophenes is minimized when one of the thiophenes possesses a fluorine substituent in the 4position. It was determined from this that the favourable sulfur-fluorine interaction overcomes the otherwise unfavorable sulfur-methyl steric clash. Optical bandgaps of $1.98 \mathrm{eV}$ for both P3HT-50F and P3HT-33F, as opposed to $1.97 \mathrm{eV}$ for P3HT-25F, and HOMO energy levels of 5.34, -5.26 and -5.24 eV, as determined from electrochemical measurements for P3HT-50F, P3HT-33F and P3HT-25F, respectively, indicated that below the 33\% fluorination point, fluorination had little effect on the electrochemical properties of P3HT. P3HT-33F also possessed the best results in field effect transistors, reaching hole mobilities of $2.89 \times 10^{-2} \mathrm{~cm}^{2}$ $\mathrm{V}^{-1} \mathrm{~s}^{-1}$. Despite the lowering of the HOMO levels and the improved on/off current ratios, the overall OFET performances of highly regioregular P3HT are still better than those of their regioregular, evenly fluorinated counterparts.

\section{Acknowledgements}

The authors acknowledge the Natural Sciences and Engineering Research Council of Canada (NSERC) and the Canadian Institute for Advanced Research (CIFAR) for their support. J. T. B. thanks the NSERC for a Canada Graduate Scholarship, S. A. L. thanks the U.S. Department of Energy Energy Efficiency and Renewable Energy postdoctoral fellowship (Solar Energy program) for funding and J. de G. S. thanks the Brazilian Coordination for the Improvement of 
Higher Education Personnel (CAPES) (Program number: Julio de Goes Soares/88888.934050/2014-00). A.A.-G. acknowledges support from the Department of Energy Basic, Energy Sciences Program in Theory and Modeling under contract DE-SC0015959. J. T. B. thanks Rodica Neagu Plesu for help with certain polymer characterization methods.

\section{Supporting Information}

Additional figures, NMR spectra, details of synthesis, computational data, MALDI-ToF data, fabrication methods for the preparation of OTFTs and device output and transfer curves.

\section{References}

1. Okamoto, T.; Nakahara, K.; Saeki, A.; Seki, S.; Oh, J. H.; Akkerman, H. B.; Bao, Z.; Matsuo, Y., Aryl-Perfluoroaryl Substituted Tetracene: Induction of Face-to-Face $\pi-\pi$ Stacking and Enhancement of Charge Carrier Properties. Chem. Mater. 2011, 23 (7), 1646-1649.

2. Stuart, A. C.; Tumbleston, J. R.; Zhou, H.; Li, W.; Liu, S.; Ade, H.; You, W., Fluorine Substituents Reduce Charge Recombination and Drive Structure and Morphology Development in Polymer Solar Cells. J. Am. Chem. Soc. 2013, 135 (5), 1806-1815.

3. Chen, H.-Y.; Hou, J.; Zhang, S.; Liang, Y.; Yang, G.; Yang, Y.; Yu, L.; Wu, Y.; Li, G., Polymer solar cells with enhanced open-circuit voltage and efficiency. Nature Photon. 2009, 3 (11), 649-653. 
4. Liang, Y.; Feng, D.; Wu, Y.; Tsai, S.-T.; Li, G.; Ray, C.; Yu, L., Highly Efficient Solar Cell Polymers Developed via Fine-Tuning of Structural and Electronic Properties. J. Am. Chem. Soc. 2009, 131 (22), 7792-7799.

5. Koster, L. J. A.; Mihailetchi, V. D.; Blom, P. W. M., Ultimate efficiency of polymer/fullerene bulk heterojunction solar cells. Appl. Phys. Lett. 2006, 88 (9), 093511.

6. Yum, S.; An, T. K.; Wang, X.; Lee, W.; Uddin, M. A.; Kim, Y. J.; Nguyen, T. L.; Xu, S.; Hwang, S.; Park, C. E.; Woo, H. Y., Benzotriazole-Containing Planar Conjugated Polymers with Noncovalent Conformational Locks for Thermally Stable and Efficient Polymer Field-Effect Transistors. Chem. Mater. 2014, 26 (6), 2147-2154.

7. Nguyen, T. L.; Choi, H.; Ko, S. J.; Uddin, M. A.; Walker, B.; Yum, S.; Jeong, J. E.; Yun, M. H.; Shin, T. J.; Hwang, S.; Kim, J. Y.; Woo, H. Y., Semi-crystalline photovoltaic polymers with efficiency exceeding $9 \%$ in a [similar] $300 \mathrm{~nm}$ thick conventional single-cell device. Energ. Environ. Sci. 2014, 7 (9), 3040-3051.

8. Leclerc, N.; Chávez, P.; Ibraikulov, O.; Heiser, T.; Lévêque, P., Impact of Backbone Fluorination on $\pi$-Conjugated Polymers in Organic Photovoltaic Devices: A Review. Polymers 2016, 8 (1), 11 .

9. El Kassmi, A.; Fache, F.; Lemaire, M., Poly(3-fluorothiophene). J. Electroanal. Chem. 1994, 373 (1), 241-244.

10. Sakamoto, Y.; Komatsu, S.; Suzuki, T., Tetradecafluorosexithiophene: The First Perfluorinated Oligothiophene. J. Am. Chem. Soc. 2001, 123 (19), 4643-4644.

11. Heeney, M.; Farrand, L.; Giles, M.; Thompson, M.; Tierney, S.; Shkunov, M.; Sparrowe, D.; McCullouch, I. U.S. Patent 6676857 B2, 2004. 
12. Gohier, F.; Frère, P.; Roncali, J., 3-Fluoro-4-hexylthiophene as a Building Block for Tuning the Electronic Properties of Conjugated Polythiophenes. J. Org. Chem. 2013, 78 (4), 1497-1503.

13. Koo, B.; Sletten, E. M.; Swager, T. M., Functionalized Poly(3-hexylthiophene)s via Lithium-Bromine Exchange. Macromolecules 2015, 48 (1), 229-235.

14. Fei, Z.; Boufflet, P.; Wood, S.; Wade, J.; Moriarty, J.; Gann, E.; Ratcliff, E. L.; McNeill, C. R.; Sirringhaus, H.; Kim, J.-S.; Heeney, M., Influence of Backbone Fluorination in Regioregular Poly(3-alkyl-4-fluoro)thiophenes. J. Am. Chem. Soc. 2015, 137 (21), 6866-6879.

15. Homyak, P. D.; Liu, Y.; Harris, J. D.; Liu, F.; Carter, K. R.; Russell, T. P.; Coughlin, E. B., Systematic Fluorination of P3HT: Synthesis of P(3HT-co-3H4FT)s by Direct Arylation Polymerization, Characterization, and Device Performance in OPVs. Macromolecules 2016, 49 (8), 3028-3037.

16. Hoth, C. N.; Choulis, S. A.; Schilinsky, P.; Brabec, C. J., On the effect of poly(3hexylthiophene) regioregularity on inkjet printed organic solar cells. J. Mater. Chem. 2009, 19 (30), 5398-5404.

17. Gao, Y.; Zhang, X.; Tian, H.; Zhang, J.; Yan, D.; Geng, Y.; Wang, F., High Mobility Ambipolar Diketopyrrolopyrrole-Based Conjugated Polymer Synthesized Via Direct Arylation Polycondensation. Adv. Mater. 2015, 27 (42), 6753-6759.

18. Bura, T.; Blaskovits, J. T.; Leclerc, M., Direct (Hetero)arylation Polymerization: Trends and Perspectives. J. Am. Chem. Soc. 2016, 138 (32), 10056-10071.

19. Koch, F. P. V.; Smith, P.; Heeney, M., "Fibonacci’s Route” to Regioregular Oligo(3hexylthiophene)s. J. Am. Chem. Soc. 2013, 135 (37), 13695-13698. 
20. Smith, K.; Barratt, M. L., Highly Selective 5-Substitution of 3-Methylthiophene via Directed Lithiation. J. Org. Chem. 2007, 72 (3), 1031-1034.

21. Loewe, R. S.; Khersonsky, S. M.; McCullough, R. D., A Simple Method to Prepare Head-to-Tail Coupled, Regioregular Poly(3-alkylthiophenes) Using Grignard Metathesis. Adv. Mater. 1999, 11 (3), 250-253.

22. Loewe, R. S.; Ewbank, P. C.; Liu, J.; Zhai, L.; McCullough, R. D., Regioregular, Headto-Tail Coupled Poly(3-alkylthiophenes) Made Easy by the GRIM Method: Investigation of the Reaction and the Origin of Regioselectivity. Macromolecules 2001, 34 (13), 4324-4333.

23. Miyakoshi, R.; Yokoyama, A.; Yokozawa, T., Catalyst-Transfer Polycondensation. Mechanism of Ni-Catalyzed Chain-Growth Polymerization Leading to Well-Defined Poly(3hexylthiophene). J. Am. Chem. Soc. 2005, 127 (49), 17542-17547.

24. Pouliot, J.-R.; Wakioka, M.; Ozawa, F.; Li, Y.; Leclerc, M., Structural Analysis of Poly(3-hexylthiophene) Prepared via Direct Heteroarylation Polymerization. Macromol. Chem. Phys. 2016, 217 (13), 1493-1500.

25. Xiao, S. S.; Qiu, C.; Qiu, C. X. U.S. Patent 6894145 B2, 2005.

26. Winter, J. D.; Deshayes, G.; Boon, F.; Coulembier, O.; Dubois, P.; Gerbaux, P., MALDIToF analysis of polythiophene: use of trans-2-[3-(4-t-butyl-phenyl)-2-methyl- 2propenylidene]malononitrile_-DCTB—as matrix. J. Mass Spectrom. 2011, 46 (3), 237-246.

27. Liu, J.; Loewe, R. S.; McCullough, R. D., Employing MALDI-MS on Poly(alkylthiophenes): Analysis of Molecular Weights, Molecular Weight Distributions, EndGroup Structures, and End-Group Modifications. Macromolecules 1999, 32 (18), 5777-5785. 28. Komber, H.; Senkovskyy, V.; Tkachov, R.; Johnson, K.; Kiriy, A.; Huck, W. T. S.; Sommer, M., Ring Walking versus Trapping of Nickel(0) during Kumada Catalyst Transfer 
Polycondensation Using Externally Initiated Electron-Accepting Thiophene-BenzothiadiazoleThiophene Precursors. Macromolecules 2011, 44 (23), 9164-9172.

29. Holdcroft, S., Determination of molecular weights and Mark-Houwink constants for soluble electronically conducting polymers. J. Polym. Sci., Part B: Polym. Phys. 1991, 29 (13), 1585-1588.

30. Kiriy, A.; Senkovskyy, V.; Sommer, M., Kumada Catalyst-Transfer Polycondensation: Mechanism, Opportunities, and Challenges. Macromol. Rapid Commun. 2011, 32 (19), 15031517.

31. Kim, J.-S.; Kim, J.-H.; Lee, W.; Yu, H.; Kim, H. J.; Song, I.; Shin, M.; Oh, J. H.; Jeong, U.; Kim, T.-S.; Kim, B. J., Tuning Mechanical and Optoelectrical Properties of Poly(3hexylthiophene) through Systematic Regioregularity Control. Macromolecules 2015, 48 (13), 4339-4346.

32. Jackson, N. E.; Savoie, B. M.; Kohlstedt, K. L.; Olvera de la Cruz, M.; Schatz, G. C.; Chen, L. X.; Ratner, M. A., Controlling Conformations of Conjugated Polymers and Small Molecules: The Role of Nonbonding Interactions. J. Am. Chem. Soc. 2013, 135 (28), 1047510483.

33. Li, Z.; Lin, H.; Jiang, K.; Carpenter, J.; Li, Y.; Liu, Y.; Hu, H.; Zhao, J.; Ma, W.; Ade, H.; Yan, H., Dramatic performance enhancement for large bandgap thick-film polymer solar cells introduced by a difluorinated donor unit. Nano Energy 2015, 15, 607-615.

34. Kohn, P.; Huettner, S.; Komber, H.; Senkovskyy, V.; Tkachov, R.; Kiriy, A.; Friend, R. H.; Steiner, U.; Huck, W. T. S.; Sommer, J.-U.; Sommer, M., On the Role of Single Regiodefects and Polydispersity in Regioregular Poly(3-hexylthiophene): Defect Distribution, Synthesis of 
Defect-Free Chains, and a Simple Model for the Determination of Crystallinity. J. Am. Chem. Soc. 2012, 134 (10), 4790-4805.

35. Zhang, L.; Colella, N. S.; Liu, F.; Trahan, S.; Baral, J. K.; Winter, H. H.; Mannsfeld, S. C. B.; Briseno, A. L., Synthesis, Electronic Structure, Molecular Packing/Morphology Evolution, and Carrier Mobilities of Pure Oligo-/Poly(alkylthiophenes). J. Am. Chem. Soc. 2013, 135 (2), 844-854.

36. Perepichka, I. F.; Perepichka, D. F., Handbook of Thiophene-based Materials: Applications in Organic Electronics and Photonics. John Wiley and Sons, Ltd. : 2009; Vol. 1, p 417.

37. McCullough, R. D., The Chemistry of Conducting Polythiophenes. Adv. Mater. 1998, 10 (2), 93-116.

38. Trznadel, M.; Pron, A.; Zagorska, M.; Chrzaszcz, R.; Pielichowski, J., Effect of Molecular Weight on Spectroscopic and Spectroelectrochemical Properties of Regioregular Poly(3-hexylthiophene). Macromolecules 1998, 31 (15), 5051-5058. 